\title{
CapablePtrs: Securely Compiling Partial Programs using the Pointers-as-Capabilities Principle
}

\author{
Akram El-Korashy ${ }^{1}$, Stelios Tsampas ${ }^{2}$, Marco Patrignani ${ }^{3}$, Dominique Devriese ${ }^{4}$, Deepak Garg ${ }^{1}$, and Frank Piessens ${ }^{2}$ \\ ${ }^{1}$ MPI-SWS, Germany; email: elkorashy@mpi-sws.org,dg@mpi-sws.org \\ ${ }^{2}$ imec-Distrinet, KU Leuven, Belgium; email name.surname@cs.kuleuven.be \\ ${ }^{3}$ Stanford University, USA and CISPA, Germany; email mp@cs.stanford.edu \\ ${ }^{4}$ Vrije Universiteit Brussel, Belgium; email dominique.devriese@vub.be
}

\begin{abstract}
Capability machines such as $C H E R I$ provide memory capabilities that can be used by compilers to provide security benefits for compiled code (e.g., memory safety). The C to $C H E R I$ compiler, for example, achieves memory safety by following a principle called "pointers as capabilities" $(P A C)$.

Informally, $P A C$ says that a compiler should represent a source language pointer as a machine code capability. But the security properties of $P A C$ compilers are not yet well understood. We show that memory safety is only one aspect, and that $P A C$ compilers can provide significant additional security guarantees for partial programs: the compiler can provide guarantees for a compilation unit, even if that compilation unit is later linked to attacker-controlled machine code.

This paper is the first to study the security of $P A C$ compilers for partial programs formally. We prove for a model of such a compiler that it is fully abstract. The proof uses a novel proof technique (dubbed $\operatorname{Tr} I C L$, read trickle), which is of broad interest because it reuses and extends the compiler correctness relation in a natural way, as we demonstrate.

We implement our compiler on top of the $C H E R I$ platform and show that it can compile legacy $C$ code with minimal code changes. We provide performance benchmarks that show how performance overhead is proportional to the number of crosscompilation-unit function calls.
\end{abstract}

\section{INTRODUCTION}

\section{A. Capability machines and pointers-as-capabilities}

In a conventional computer, memory locations are addressed using integers. There, a store instruction such as sw $\$ r 2 \mathrm{n}(\$ r 0)$ interprets the contents of register $\$ r 0$ as a virtual address (which is an integer), and adds $n$ to it to obtain the (integer) address where to store the contents of $\$ r 2$. In other words, the memory model of a conventional computer is essentially an array of integers indexed by integers. Instead, in a capability machine (i.e., a capability-based computer), memory locations are addressed using a capability. Capabilities are a separate type of run-time values that carry more information than just a memory address. They also contain bounds information indicating a section of memory that can be accessed using this capability, and possibly also other information such as access permissions. The store instruction csw $\$ r 2 \mathrm{n}(\$ c 0)$ now uses a capability register $\$ \mathrm{c} 0$, and the machine will check that the store performed by this instruction is within bounds, and is compliant with the permissions associated with the capability in $\$ c 0$. If $\mathrm{n}$ is too big, or if $\$ \mathrm{c} 0$ only has permission for reading, the store instruction will fail with an exception. The hardware also implements run-time type checks to ensure that integers and capabilities can not be confused, for instance capabilities are stored in separate register banks, and capabilities are tracked in memory by tagging memory locations that contain a capability. Hence, capability machines implement a more structured memory model where (somewhat simplified) memory is a collection of independent integerindexed arrays, containing integers or capabilities, and every capability gives access to a contiguous segment of one of those arrays. This more structured memory model can be used to implement fine-grained memory protection, and has the potential to provide protection against many software bugs.

Unfortunately, this support for memory protection is only useful if high-level software can use it, and manually modifying large existing code bases to adopt this memory protection is not practically feasible. Hence, ideally, compilers should handle this automatically. Thus, capability machines should be designed to make it easy for compilers of existing languages (like $\mathrm{C}$ ) to use the additional support for memory protection. A recent example of such a machine is CHERI, a mature capability machine implementation that has its own FreeBSD version and C compiler [1, 2, 3, 4, 5]. Many key design choices in CHERI were made to facilitate the use of memory protection in existing, large code bases. Specifically, $C H E R I$ supports the pointers-as-capabilities $(P A C)$ principle, which intuitively dictates that a compiler should represent a source-level pointer as a target-level capability. To make this convenient, a CHERI capability contains (among other things): base and length addresses, and an offset relative to the base address [5]. Such a capability represents a pointer pointing to the address base + offset, and that is valid for accessing (using indexing or pointer arithmetic) addresses in the range [base, base + length). Pointer arithmetic can be implemented by manipulating the offset.

The following example illustrates how a compiler can map C pointers to such machine-level capabilities. ${ }^{1}$

\footnotetext{
1 As a typesetting convention, we use a blue, sans-serif font for source language elements and an orange, bold font for target language ones. Elements common to both languages are typeset in a black, italic font.
} 
Example 1 (From C Pointers to CHERI Capabilities). The $C$ compilation unit (below on the left) declares two modulescoped variables and defines a function $f()$ using one of these variables. The assembly pseudocode (below on the right) shows how a PAC compiler could translate the body of $f()$.

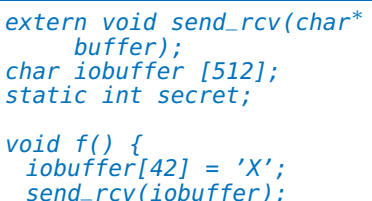

The default data capability register $\$$ ddc contains a capability for the global data section. The compiler knows that the variable iobuffer occupies the first 512 bytes of that global data section. Hence, the first instruction (CSetLen, set length of a capability) loads in register $\$ c 1$ a copy of $\$ d d c$ but with the length field reduced to 512. The next two instructions implement the assignment instruction of $f()$. Note that an out-of-bounds access would be trapped by the hardware. The final call instruction implements the function call in $f$ () (assuming a calling convention where the parameter is passed in register $\$ \subset 1)$. Note that also all accesses to iobuffer performed in send_rcv will be bounds-checked, since the capability representing the pointer passed to send_rcv carries the bounds information.

\section{B. Security properties of a PAC compiler}

Clearly, a $P A C$ compiler provides security benefits. But what security properties does it provide exactly?

First, the compiler provides spatial memory safety. Since the bounds meta-data for a pointer is stored together with the pointer address in a single capability value, it is straightforward to implement a bounds-checking compiler. For instance, an out-of-bounds access to iobuffer in the example above will not access the secret variable, but will fail instead. It is in principle also possible to have the compiler provide temporal memory safety. The compiler should then emit code to revoke a capability when the corresponding memory region is freed, but there are many challenges to do this efficiently. For instance, revocation can be implemented by searching all reachable memory and zeroing out any remaining capabilities for the memory region to be freed, but this is clearly inefficient, in particular for revoking stack allocated memory. Several papers study approaches to efficiently achieve temporal memory safety on a capability machine, both for stack $[6,7,8]$ and heap memory [9], but it is fair to say that achieving efficient temporal memory safety for $\mathrm{C}$ is still an open problem. Hence, we leave it out of scope for this paper.

But memory safety is not the full story. A $P A C$ compiler can provide stronger security guarantees. Consider again the example above, but now under the assumption that the external send_rcv function is not implemented in $\mathrm{C}$, but directly in assembly code. Now, we lose the guarantee that send_rcv can not access the secret variable. An assembly level implementation can directly access $\$ d d c$. Hence, memory safety is only guaranteed for complete programs: if all code in a program is compiled by the $P A C$ compiler, then all out-of-bounds accesses will be trapped.

Since capability machines are designed to guarantee unforgeability of capabilities even at machine code level, a $P A C$ compiler can provide stronger guarantees than spatial memory safety for complete programs. The main contribution of this paper is that we show how a $P A C$ compiler can provide strong security guarantees for partial programs. In particular, for the example above, the compiler we discuss in this paper provides the guarantee that secret is inaccessible, even if the external function is implemented in hand-crafted assembly code.

In order to achieve non-trivial security guarantees for partial programs, the target capability machine needs to support a mechanism to define separate protection domains within a single process. For instance, CHERI provides support for object capabilities [3, 4]. This makes it possible to put different program parts in separate protection domains. CHERI mainly uses the mechanism of object capabilities to compartmentalize programs: it offers an API to programmers to run parts of a program in a sandbox, a protection domain with reduced privileges. The current CHERI compiler, however, does not make direct use of the object capability mechanism: it can only be used through the provided API by the programmer who has to define and set up sandboxes. The $P A C$ compiler we propose in this paper, on the other hand, will automatically set up a separate protection domain for every compilation unit. Doing so allows the compiler to provide strong security guarantees for partial programs. In addition to the guarantees for partial programs against hand-crafted assembly, this automatic compartmentalization can significantly reduce the potentially dangerous impact of bugs in one compilation unit on the operation of other compilation units, again without any programmer effort.

\section{Summary of our results}

We study the security guarantees that a $P A C$ compiler can provide for partial programs. We prove a security theorem about a simple model of a compartmentalizing $P A C$ compiler, and discuss its implications. We implement the compiler for full $\mathrm{C}$ on top of the existing CHERI compiler, and we evaluate the performance cost as well as the compatibility of the compiler with existing code.

Our formulation and proof of the security guarantees of $P A C$ models $P A C$ as a compiler from a very simple imperative source language with pointers, to a capability machine with memory capabilities and a very basic form of protection domains/object capabilities. The security theorem we prove takes the form of a full abstraction result [10], which intuitively means that it is valid for programmers to reason about the security of their program parts in the source language. If a program part has a security property (such as the confidentiality of the secret variable in the example) that is valid according to the source language semantics (i.e., under 
any interaction of the program part with other source language program parts), then that property remains valid also if the compiled program part is linked with attacker-crafted machine code.

This is a very strong security property, and we only prove it under some (reasonable) restrictions. First, some syntactic restrictions need to be imposed on the (attacker-provided) machine code. These restrictions can be implemented as a code verification step by the linker. The main restriction we rely on is that machine code should not directly access the program counter capability, a restriction that is easy to check at link time, and that guarantees that machine code can not confuse a program part by providing it a code capability where it expects a data capability.

Second, to make source-code-level reasoning sound even when linking to machine code, we need to make some of the machine-code level power of the attacker also available at source code level, essentially forcing the programmer to take this into account when reasoning about his program part at source code level. Consider for instance the following example:

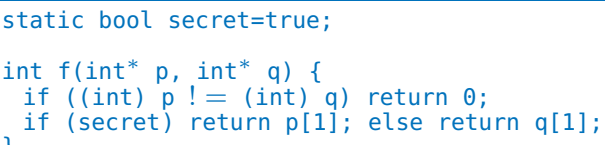

Function $f()$ first tests whether its two argument pointers are equal addresses. This equality implies that $p$ [1] and $q$ [1] always evaluate to the same value (or both fail). Hence $f()$ does not leak information about secret.

However, a machine code level adversary can call $f()$ with two capabilities that point to the same address but have different bounds information. In that case accessing $p$ [1] could fail, while q[1] returns a value, and f() does leak information about secret. Hence, to prove our theorem, we have to extend the source language to make pointers carry bounds information. This essentially makes explicit exactly what aspects of the target language programmers need to take into account to reason soundly about partial source programs.

Full abstraction $(F A)$ theorems are known to be hard to prove, and the same goes for our theorem. Our proof adopts a novel proof technique called $\operatorname{Tr} I C L$ (read, trickle) that relies on trace semantics for both source and target languages. While inspired by existing $F A$ proofs, this technique is both novel and of broad interest for secure compilation since it simplifies trace-based proofs that are very common for secure compilation results (as we describe later on). $\operatorname{Tr} I C L$ simplifies tracebased proofs by providing a general technique for re-using the simulation relation that is built for compiler correctness, and for extending it into an alternating simulation relation for the trace-based security proof.

In summary, this paper makes the following contributions:

- it is the first to state and prove the security properties of a $P A C$ compiler for partial programs. To do so, the paper makes substantial technical contributions:
- the definition of a sound and complete trace semantics for a C-like language (Section II-A) and for a language with capabilities (Section II-B). Both languages feature a memory model that allows fine-grained memory sharing, which makes our results more interesting, our proofs more challenging and, in turn, our proof techniques more widely-applicable;

- the definition of a compiler between the aforementioned languages that embodies the pointers-ascapabilities design (Section III);

- a proof that said compiler is fully abstract. In this proof, we use a novel proof technique called $\operatorname{Tr} I C L$ (Section IV);

- it reports on an implementation of that compiler on top of the C-to-CHERI compiler and benchmarks its efficiency and compatibility with existing $\mathrm{C}$ code (Sections V and VI).

Our contributions are followed by a discussion of our model's limitations (Section VII) and related work (Section VIII).

Most of the formalisation presented in this paper is massaged for presentation, and many auxiliary lemmas as well as proofs are omitted; the interested reader will find the full formal details at https://github.com/capable-ptrs/tech-report. The implementation of our compiler and the related benchmarks will be made available publicly on publication of this paper.

\section{Models OF THE SOURCE AND TARGET LANGUAGES}

In this section, we introduce our models of the source and target languages: ImpMod (Section II-A) and CHERIExpress (Section II-B). But first, we explain notation that is used throughout the paper including notation for contextual equivalence.

Given execution states $s, s^{\prime}$, and a small-step relation $\rightarrow$ (with a reflexive transitive closure $\rightarrow^{*}$ ), we denote by $s \rightarrow s^{\prime}$ the judgment that state $s$ executes and transitions into state $s^{\prime}$. Such a state $s$ is not stuck. If on the other hand, no $s^{\prime}$ exists such that $s \rightarrow s^{\prime}$, then we say $s$ is stuck. In our models, for simplicity, we treat errors the same way we do stuck states, i.e., we do not explicitly distinguish the event of an exception with any special error state. An execution that reaches a stuck state or that never terminates is referred to as diverging.

Programs and initial \& terminal states: A program is a list of modules, and a module is a list of functions. Linking of a pair of programs is denoted by $\ltimes$. We define linking to be non-symmetric because it makes our security proof easier (In Section VII, we discuss the reason for this non-symmetry). For the sake of our theorem, we will distinguish two parts $\mathfrak{C}$ and $p$ of a linked program $\mathfrak{C} \ltimes p$ as the context and the program, suggesting that the latter is the program of interest because it is the program that is or has been translated by our compiler. But notice that each of the program of interest and the context may themselves consist of more than one module (i.e., more than one compilation unit). Our results do not assume any restrictions on the number of modules that the program or the 
context may consist of. As usual, only whole programs with a main function can execute correctly.

The initial state of a program $p$ is denoted by init $(p)$. A state $s$ is called a terminal state when it satisfies the judgment $\vdash_{t} s$.

If the execution of a program of interest $p$ in a certain context $\mathfrak{C}$ reaches a terminal state, then we say the execution converges (or instead say the program converges), and we denote this fact by $\mathfrak{C}[p] \Downarrow$ which is shorthand for the following:

$$
\exists s \text {. init }(\mathfrak{C} \ltimes p) \rightarrow^{*} s \wedge \vdash_{t} s
$$

Now with the notation for convergence in hand, we define contextual equivalence of programs as follows:

Definition 1 (Contextual equivalence of programs). $p_{1} \simeq_{\operatorname{ctx}} p_{2} \stackrel{\text { def }}{=} \forall \mathfrak{C} . \mathfrak{C}\left[p_{1}\right] \Downarrow \Longleftrightarrow \mathfrak{C}\left[p_{2}\right] \Downarrow$

Two programs $p_{1}$ and $p_{2}$ whose termination behaviors (i.e., whether they converge or not) always agree whenever linked and executed with the same context are contextually equivalent $(\simeq \operatorname{ctx})$.

\section{A. ImpMod: An imperative language with modules, arrays,} and pointers

For simplicity, and in order to focus on the "pointers to capabilities" aspect of the translation, we design ImpMod to be low-level enough so that we do not need to worry about extra correctness arguments that are otherwise pretty standard. For example, ImpMod features only unstructured control flow in the form of a JumplfZero instruction. But it still features functions and modules.

In fact, modules are crucial to implementing the "pointers to capabilities" translation. They are the scope of module-global variables and they are also used as a unit of isolation; i.e., 1) every module gets its own data stack on which it stores the frames of alive calls to its functions, and 2) every function inside a module can access all the module-global variables, whereas any function external to the module can only call the module's functions but is by default not allowed to access its module-global variables.

For example, in Listing II.1 below, we have an example module (Main) where variables iobuffer and secret (lines 4 \& 5) are both module-global variables.

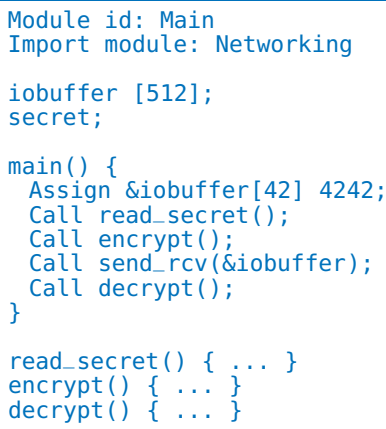

Listing II.1: Example ImpMod module.
All of the main function ${ }^{2}$ and the secret-handling functions: read_secret, encrypt and decrypt are defined in the same module (Main), and thus can each access both variables (iobuffer and secret). The function send_rov in contrast is external: it is defined in the Networking module which is presumably untrusted. In line 11, the untrusted Networking module gains access to the iobuffer. The Networking module, however, will not gain access to secret through \&iobuffer-so long as the other trusted functions read_secret and encrypt also make sure to not copy (a pointer to) the secret to the iobuffer. Any attempt to increment and access the pointer \&iobuffer beyond the bounds of the array will get stuck. To understand how we model this bounds check, we introduce the expression semantics and the memory model of ImpMod.

1) Expressions and memory model of ImpMod: Expressions are denoted with e.

$$
\begin{aligned}
\text { e }::=\mathbb{Z} & |\operatorname{VarlD}| \text { e } \oplus \text { e } \mid \text { e }[e]|\& \operatorname{VarlD}| \& e[e] \mid *(e) \\
\mid & \operatorname{start}(e) \mid \text { end }(e)|\operatorname{offset}(e)| \operatorname{capType}(e) \\
\mid & \operatorname{limRange}(e, e, e)
\end{aligned}
$$

Base expressions are integers $\mathbb{Z}$ and variable identifiers VarlD. On top of the base expressions, arithmetic expressions are generically denoted with e $\oplus$ e. Pointer and array expressions are: 1) the array-offset expression e[e], 2) the ampersand operator of the forms \&VarlD and \&e[e], and 3) the star operator $*(\mathrm{e})$. Moreover, there are low-level expressions that are necessary for reflecting the target memory model in the source language (as mentioned in Section I-B): four getters: start(e), end(e), offset(e), and capType(e); and a setter: limRange $(e, e, e)$. These low-level expressions operate on the capability-based representation of memory addresses that we explain next.

Addresses in ImpMod are represented as capabilities. Thus, built into the memory model is a type Cap $\stackrel{\text { def }}{=}\{\kappa, \delta\} \times \mathbb{Z} \times \mathbb{Z} \times \mathbb{Z}$ for capabilities that is distinct from integers $\mathbb{Z}$. Hence, run-time values $V$ are integers $\mathbb{Z}$ or capabilities Cap. And therefore, a memory Mem : $\mathbb{Z} \stackrel{\text { fin }}{\longrightarrow}$ of a program's execution state in ImpMod can contain capability values. The first field of the capability value indicates its type: code $(\kappa)$ or data $(\delta)$. The getter of this field is the expression capType. The next three (integer) fields are respectively the start, end, and offset of a capability. The start and end identify the memory region on which this capability authorizes a (code $(\kappa)$ or data $(\delta))$ access operation. The offset designates the one address at which an access operation is performed. The offset should be within range (checked by Rule Eval-star):

$$
\begin{gathered}
\text { (Eval-star) } \\
e \Downarrow(\delta \text {, st, end, off }) \\
\text { st } \leq \text { st }+ \text { off }<\text { end } \text { Mem }(\text { st }+ \text { off })=v \\
*(e) \Downarrow v
\end{gathered}
$$

For space constraints, the full expression semantics is given in the appendix in Figure 5.

\footnotetext{
${ }^{2}$ Notice in the main function on line 8 the use of variable iobuffer; the syntax for 1-values is more explicit than $\mathrm{C}$ syntax.
} 
2) Commands and execution state of ImpMod: Commands of ImpMod are denoted with Cmd:

$$
\begin{aligned}
\text { Cmd ::= } & \text { Assign } e_{\mid} e_{r} \mid \text { Alloc e e } e_{\text {size }} \mid \text { Call fid } \bar{e} \mid \text { Return } \\
& \mid \text { JumplfZero } e_{c} e_{o f f} \mid \text { Exit }
\end{aligned}
$$

An execution state $s$ of a program in ImpMod consists of the memory Mem, the stack pointers $\Phi$ of the module-local stacks, the program counter $\mathrm{pc}$, the trusted control stack stk — which is shared among all modules of a program - and the memoryallocation status represented by the next-free-address nalloc. The space for dynamic memory allocation (i.e., the heap) is also shared by all the program modules (like the trusted control stack). The semantics for allocation and assignment are given in the appendix in Figure 6.

\section{B. CHERIExpress: An imperative language with modules, and capabilities}

Our target language CHERIExpress is (like ImpMod) an imperative language with modules. However (unlike ImpMod), it does not feature variables, neither global nor local. Instead, it only features "capability registers" and integers as base expressions. With these capability registers in hand, the role of the compiler from ImpMod to CHERIExpress is to implement the operations on pointers by using operations on capability registers. The memory model (as explained already in Section II-A1 about the memory model of ImpMod) is capability based. Through this capability-based memory model and the capability registers, CHERIExpress models a higher-level and simplified version of CHERI assembly [11].

1) Expressions and commands in CHERIExpress: Expressions are denoted with e.

$$
\begin{aligned}
\text { e ::= } \mathbb{Z} \mid & \text { getddc } \mid \text { getstc } \mid \text { e } \oplus \text { e }|\operatorname{inc}(e, e)| \operatorname{deref}(e) \\
& |\operatorname{start}(e)| \operatorname{end}(e)|\operatorname{offset}(e)| \operatorname{capType}(e) \\
& \mid \operatorname{limRange}(\mathbf{e}, \mathbf{e}, \mathbf{e})
\end{aligned}
$$

No expression allows fabrication of an arbitrary capability thus exhibiting capability unforgeability. The getter expressions getddc and getstc are the only base expressions that evaluate to a capability value. Evaluation of the expressions getddc and getstc immediately results in the current value of the respective capability registers, ddc and stc. ${ }^{3}$

(Eval-ddc)

Mem, ddc, stc, pcc $\vdash$ getddc $\Downarrow$ ddc

There is a third capability register that is also part of the execution state, namely, the pcc register. It holds the program counter capability which points to and allows the execution of commands. This register is however eliminated from the possible expression forms in CHERIExpress. This elimination is a simple way of enacting in our model the prohibition (mentioned in Section I-B) on linking with contexts that

\footnotetext{
${ }^{3}$ The choice of the names of capability registers hints at their recommended usage: ddc stands for default data capability [11], and stc which we defined for CHERIExpress stands for stack capability. We use ddc as a capability on the per-module data segment, and stc on the per-module stack.
}

mention the pcc register. Commands in CHERIExpress are (modulo expressions) the same as commands in ImpMod. Thus, instead of presenting here the full operational semantics, we focus next on presenting the details of our $P A C$ compiler.

\section{ImpMod TO CHERIExpress: A POINTERS-AS-CAPABILITIES COMPILER}

Our ImpMod to CHERIExpress compiler $\llbracket \cdot \rrbracket$ is a $P A C$ compiler. In this section, we present its crucial bits, namely, the translation of ImpMod expressions to CHERIExpress expressions. The translations of commands (denoted $(\cdot 0)$ and of modules (denoted $\llbracket \cdot \rrbracket$ ), on the other hand, are not surprising due to the closeness of the source and target languages.

The translation of expressions $\chi \cdot \int: e \rightarrow e$, whose excerpts are presented below, is indexed by the syntactic information fid, modID (function id and module id) giving the scope of the expression being translated, and $\beta$ giving layout and bounds of variables.

$$
\begin{aligned}
& 2 \mathrm{z} \int_{-} \stackrel{\text { def }}{=} \mathrm{z} \\
& \left\{\mathrm{e}_{1} \oplus \mathrm{e}_{2} \int \mathrm{fid}, \mathrm{modID}, \beta \stackrel{\text { def }}{=} \chi \mathrm{e}_{1} \int \mathrm{fid}, \text { modID }, \beta \oplus \chi \mathrm{e}_{2} \int \mathrm{fid}, \bmod \mathrm{D}, \beta\right. \\
& \text { ¿\&vid } \int_{-}, \text {modID }, \beta \stackrel{\text { def }}{=} \operatorname{limRange}(\mathrm{ddc} \\
& \operatorname{start}(\mathbf{d d c})+\mathrm{st}, \operatorname{start}(\mathrm{ddc})+\text { end }) \\
& \text { when } \beta(\text { vid }, \perp \text {, modID })=(\text { st, end }) \\
& \text { ¿\&vid } \int_{\text {fid }, \text { modID }, \beta} \stackrel{\text { def }}{=} \operatorname{limRange}(\text { stc }, \text { st }+\operatorname{start}(\text { stc }) \\
& + \text { offset(stc), end }+\operatorname{start}(\text { stc }) \\
& +\operatorname{offset}(\mathrm{stc})) \\
& \text { when } \beta(\text { vid, fid, modID })=(\text { st, end }) \\
& \text { ¿vid } \int_{\text {fid, mid, } \beta} \stackrel{\text { def }}{=} \operatorname{deref}\left(\eta \& \text { vid } \int_{\text {fid, } \text { mid }, \beta}\right) \\
& \eta \& \mathrm{e}_{\mathrm{arr}}\left[\mathrm{e}_{\mathrm{off}}\right] \int \mathrm{fid}, \mathrm{mid}, \beta \stackrel{\text { def }}{=} \operatorname{inc}\left(\chi \& \mathrm{e}_{\mathrm{arr}} \int \mathrm{fid}, \mathrm{mid}, \beta, \chi \mathrm{e}_{\mathrm{off}} \int \mathrm{fid}, \mathrm{mid}, \beta\right) \\
& \left\langle e_{\text {arr }}\left[e_{\text {off }}\right] \int_{\text {fid, mid }, \beta} \stackrel{\text { def }}{=} \operatorname{deref}\left(\chi \& e_{\text {arr }}\left[e_{\text {off }}\right] \int \text { fid, mid, } \beta\right)\right.
\end{aligned}
$$

Translating expressions start, end, offset, capType, and limRange is straightforward and, similar to $\left\langle e_{1} \oplus e_{2} \int\right.$, they are homomorphisms (as expected [12]). Drawing on the translation of expressions we come again to the ImpMod program of Listing II. 1 where Line 8 is translated as shown in Example 2:

Example 2 (Translation line 8 from Listing II.1).

$$
\begin{aligned}
& (\text { Assign \&(iobuffer[42]) 4242) } \\
= & \text { Assign } 2 \&(\text { iobuffer }[42]) \int ~ 24242 \int \\
= & \text { Assign inc }\left(2 \& \text { iobuffer } \int, 42\right) 4242 \\
= & \text { Assign inc }(\operatorname{limRange}(\text { ddc, } \operatorname{start}(\text { ddc })+0, \\
& \quad \text { start }(\text { ddc })+512), 42) 4242
\end{aligned}
$$

Note that the compiler uses the bound information that is given in the text of the program in the declaration of the array (Line 4) to introduce explicit curbing (using limRange) of the ddc capability so that the resulting capability is of the same size as the declared array size (512) and not bigger, a curbing that in turn ensures that the assignment succeeds only 
when the offset is within the declared bounds of the array. In Line 8 , we could already ensure the same by trivial static analysis. But more crucially, when static analysis is not feasible, this automatic curbing introduced by the compiler ensures that the run-time check that CHERIExpress provides is done against the correct bounds.

In Line 11, the main function passes a pointer to an external untrusted function (here no static analysis is feasible unless the external function were trusted). Example 3 shows how to translate Line 11.

Example 3 (Translation line 11 from Listing II.1).

$$
\begin{aligned}
& \text { (Call send_rcv(\&iobuffer })) \\
= & \text { Call send_rcv }(\operatorname{limRange}( \\
& \text { ddc, start }(\text { ddc })+0, \operatorname{start}(\text { ddc })+512))
\end{aligned}
$$

Again, curbing the ddc capability is achieved by means of the limRange expression and it is crucial for the security proof. Without such curbing, we would not be able to prove full abstraction of our compiler. In fact without it, the untrusted send_rcv function will have access to the secret when by definition of the semantics of ImpMod it should not.

We next explain the end-to-end security guarantee that our $P A C$ compiler provides.

\section{PROVING THE COMPILER IS SECURE}

This section discusses $F A$, the formal criterion we prove to demonstrate that our compiler is secure (Section IV-A). Then it presents a high-level overview of the proof (Section IV-B). The proof relies on the definition of auxiliary trace semantics (Section IV-C), and on a novel proof technique $(\operatorname{Tr} I C L)$ that allows extending the compiler-correctness relation into an alternating simulation relation that is indexed by a trace (Section IV-D).

\section{A. Fully abstract compilation, formally}

Our compiler $\llbracket \cdot \rrbracket$ is provably secure. The formal definition that we choose for compiler security is standard [10, 13], namely, full abstraction. A compiler is fully abstract when it both reflects and preserves contextual equivalence.

Theorem 1 (The compiler $\llbracket \cdot \rrbracket$ is fully abstract).

(i) The compiler $\llbracket \cdot \rrbracket$ reflects contextual equivalence: $\forall p_{\mathrm{s} 1}, \mathrm{p}_{\mathrm{s} 2} \cdot \llbracket \mathrm{p}_{\mathrm{s} 1} \rrbracket \simeq \mathrm{ctx} \llbracket \mathrm{p}_{\mathrm{s} 2} \rrbracket \Longrightarrow \mathrm{p}_{\mathrm{s} 1} \simeq_{\mathrm{ctx}} \mathrm{p}_{\mathrm{s} 2}$

(ii) The compiler $\llbracket \cdot \rrbracket$ preserves contextual equivalence: $\forall p_{\mathrm{s} 1}, \mathrm{p}_{\mathrm{s} 2} \cdot \llbracket \mathrm{p}_{\mathrm{s} 1} \rrbracket \simeq \operatorname{ctx} \llbracket \mathrm{p}_{\mathrm{s} 2} \rrbracket \Longleftarrow \mathrm{p}_{\mathrm{s} 1} \simeq_{\mathrm{ctx}} \mathrm{p}_{\mathrm{s} 2}$

The compiler reflecting contextual equivalence (condition (i)) means that whenever it produces two contextually equivalent programs $\left(\llbracket p_{s 1} \rrbracket \simeq \operatorname{ctx} \llbracket p_{s 2} \rrbracket\right)$, then the source programs themselves must also have been contextually equivalent $\left(p_{s 1} \simeq_{c t x} p_{s 2}\right)$. This condition (reflection of contextual equivalence) ensures that the transformation $\llbracket \cdot \rrbracket$ is non-trivial. A trivial compiler might compile semantically different programs to the same output program. However, it would violate condition (i) because semantically different source programs are by definition not contextually equivalent. Notice that condition (i) usually ${ }^{4}$ even follows from whole-program correctness, namely, whole-program backward simulation (together with some structural lemmas about linking and compilation). Whole-program backward simulation is a standard [14, 15] formal criterion for considering a compiler bug-free with respect to a given source semantics.

Condition (ii), on the other hand, ensures that whenever two source programs, $p_{\mathrm{s} 1}$ and $\mathrm{p}_{\mathrm{s} 2}$, are not distinguishable in the source semantics $\left(p_{s 1} \simeq_{c t x} p_{s 2}\right)$, then after compilation, they should remain indistinguishable in the target semantics $\left(\llbracket p_{s 1} \rrbracket \simeq_{c t x} \llbracket p_{s 2} \rrbracket\right)$. Loosely speaking, what this condition ensures is that no extra distinguishing power may be gained by writing distinguishing contexts in the target language (i.e., by attempting to distinguish the compiled rather than the source programs) compared to writing contexts in the source.

\section{B. Overview of the proof}

To prove that our compiler is fully abstract, we prove both the contrapositive of condition (i), and the contrapositive of condition (ii). The former follows easily from compiler correctness and its proof is fully detailed in the supplementary material. The latter, namely preservation of contextual equivalence, is depicted in Figure 1 and it follows from three main lemmas (Lemmas 1 to 3 ) that all rely on an auxiliary (and more manageable) definition of program equivalence called trace equivalence (denoted $\stackrel{\mathrm{T}}{=}$ ).

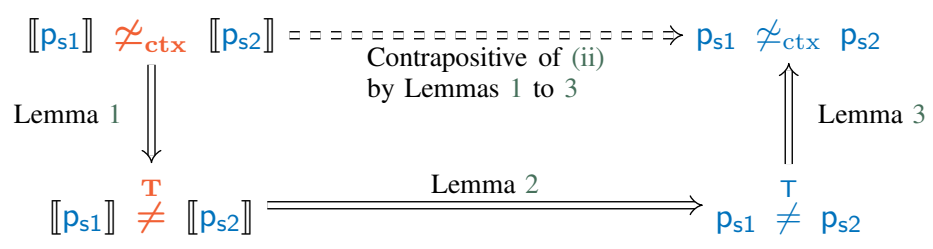

Fig. 1: Visual decomposition of our proof of (ii).

Lemma 1 (Soundness of target trace-equivalence).

$$
\forall p_{\mathrm{s} 1}, \mathrm{p}_{\mathrm{s} 2} \cdot \llbracket \mathrm{p}_{\mathrm{s} 1} \rrbracket \not \mathcal{\leftarrow}_{\mathrm{ctx}} \llbracket \mathrm{p}_{\mathrm{s} 2} \rrbracket \Longrightarrow \llbracket \mathrm{p}_{\mathrm{s} 1} \rrbracket \stackrel{\mathrm{T}}{\neq} \llbracket \mathrm{p}_{\mathrm{s} 2} \rrbracket
$$

Lemma 2 (Compilation preserves trace equivalence).

$$
\forall \mathrm{p}_{\mathrm{s} 1}, \mathrm{p}_{\mathrm{s} 2} \cdot \llbracket \mathrm{p}_{\mathrm{s} 1} \rrbracket \stackrel{\mathrm{T}}{\neq} \llbracket \mathrm{p}_{\mathrm{s} 2} \rrbracket \Longrightarrow \mathrm{p}_{\mathrm{s} 1} \stackrel{\mathrm{T}}{\neq} \mathrm{p}_{\mathrm{s} 2}
$$

Lemma 3 (Completeness of source trace-equivalence).

$$
\forall \mathrm{p}_{\mathrm{s} 1}, \mathrm{p}_{\mathrm{s} 2} \cdot \mathrm{p}_{\mathrm{s} 1} \stackrel{\mathrm{T}}{\neq} \mathrm{p}_{\mathrm{s} 2} \Longrightarrow \mathrm{p}_{\mathrm{s} 1} \neq_{\mathrm{ctx}} \mathrm{p}_{\mathrm{s} 2}
$$

The three lemmas above, put together as in Figure 1, establish that the compiler preserves contextual equivalence (the horizontal dashed arrow). One can intuitively understand them as follows:

1) Lemma 1 ensures we can abstract contextual equivalence as trace equivalence.

\footnotetext{
${ }^{4}$ Assuming the common ways of defining termination, and of defining contextual equivalence to be equi-termination under all linkable contexts
} 
2) Lemma 2 ensures (in the contrapositive) that the compiler preserves trace equivalence.

3) Lemma 3 finally ensures we can concretize trace equivalence back as contextual equivalence.

Lemma 2 is the hardest, so we dedicate Section IV-D to explaining its proof. The proofs of Lemmas 1 and 3 reuse some of the ideas introduced in Section IV-D as well. While the general approach (Figure 1) that we adopt-namely, the use of trace equivalence as a go-between in the proof-is not new [16, 17, 18, 19], we believe the elaboration here in this paper of many details (especially the trace-indexed crosslanguage ( $\operatorname{Tr} I C L)$ simulation) is a novelty of this work. The reason we come up with the $\operatorname{Tr} I C L$ simulation technique is to provide a principled way of re-using the simulation relation that is built for compiler correctness by extending it into an alternating simulation relation for the trace-based security proof (namely, the proof of Lemma 2). This re-usability advantage might spark the interest of researchers in extending state-ofthe-art correctness proofs for realistic compilers into security proofs (potentially by targeting architectures like CHERI).

\section{Trace equivalence}

The reason we define this auxiliary program equivalence called trace equivalence is to abstract over the execution steps $\rightarrow$ that are made by the target context. The way we abstract is we characterize the behavior of a program of interest $p$ by a set $\operatorname{Tr}(p)$ of informative trace prefixes $\alpha$ that capture all the possible interactions of the program $p$ with any context. Each trace prefix $\alpha$ is a finite list $\bar{\lambda}$ of labels $\lambda$. Two programs $p_{1}$ and $p_{2}$ are then trace equivalent (written $p_{1} \stackrel{\mathrm{T}}{=} p_{2}$ as noted earlier) whenever they have the same set $\operatorname{Tr}(p)$ of informative trace prefixes $(\alpha)$ :

Definition
$p_{1} \stackrel{\text { T }}{=} p_{2} \stackrel{\text { def }}{=} \forall \alpha . \alpha \in \operatorname{Tr}\left(p_{1}\right) \Longleftrightarrow \alpha \in \operatorname{Tr}\left(p_{2}\right)$

It then remains to understand how we define the set $\operatorname{Tr}(p)$ for a program $p$. To explain that, we show an excerpt of the labeled trace-step relation $\stackrel{\lambda}{\rightarrow}_{\mathrm{p}}$ that we define for our target language CHERIExpress. Note that a very similar trace step relation is defined for ImpMod programs too. The tracestep relation relates two trace states and a label $\lambda$. A trace state in CHERIExpress is written as $(s, \varsigma)$ : it extends the normal execution state s with auxiliary information $\varsigma$, which is the set of memory addresses shared so far (i.e., from the initial state and up until execution state $\mathrm{s}$ ) between the program of interest $\mathrm{p}$ and the context. This auxiliary information is used to define an informative trace label $\lambda$, which records a snapshot Mem of the entire shared memory. Trace labels $\lambda$ of both our languages have the following forms:

$$
\begin{aligned}
\lambda::=\tau|\checkmark| & \mid \operatorname{call}(\text { fid }) \bar{v} ? \text { Mem, nalloc } \\
& \mid \operatorname{call}(\text { fid }) \bar{v} ! \text { Mem, nalloc } \\
& \mid \text { ret ? Mem, nalloc } \\
& \mid \text { ret ! Mem, nalloc }
\end{aligned}
$$

1) A silent label $\tau$ is uninformative: it abstracts over any execution step that is internal to either the program or the context.

2) A termination label $\checkmark$ indicates a terminal execution state was reached. (Once a $\checkmark$ appears, it re-appears in all next trace positions.)

3) An input call label call(fid) $\bar{v}$ ? Mem, nalloc indicates that at an execution state where the shared memory was $M e m$, and the allocator status was nalloc, the context called the program's function fid with the list of values $\bar{v}$ as arguments.

4) An output call label call(fid) $\bar{v} !$ Mem, nalloc is similar except now in the opposite direction: the program called the context's function fid.

5) An input return label ret? Mem, nalloc indicates that at an execution state where the shared memory was $\mathrm{Mem}$, and the allocator status was nalloc, the context returned control to a caller function in the program.

6) An output return label ret ! Mem, nalloc is similar except the program was the one that returned control to the context.

Fig. 2: Trace semantics of CHERIExpress (Excerpts) . The trace-step relation is indexed with a program $\mathrm{p}$.

$$
\begin{aligned}
& \text { (Return-to-program) } \\
& \mathrm{s} \rightarrow \mathrm{s}^{\prime} \quad \mathrm{s} \cdot \mathrm{M}_{\mathrm{c}}(\mathrm{s} \cdot \mathrm{pcc})=\text { Return } \\
& \text { s.pcc } \not \text { dom }\left(\text { p. } \mathrm{M}_{\mathrm{c}}\right) \quad \mathrm{s}^{\prime} \cdot \mathrm{pcc} \subseteq \operatorname{dom}\left(\mathrm{p} \cdot \mathrm{M}_{\mathrm{c}}\right) \\
& \varsigma^{\prime}=\text { reachable_addresses_closure }\left(\varsigma, \mathrm{s}^{\prime} . \mathrm{Mem}\right) \\
& (\mathrm{s}, \varsigma) \stackrel{\text { ret? }\left.\mathrm{s}^{\prime} \cdot \mathrm{Mem}\right|_{\varsigma^{\prime}}, \mathrm{s} \text {.nalloc }}{\mathrm{p}}\left(\mathrm{s}^{\prime}, \varsigma^{\prime}\right) \\
& \text { (Call-to-program-silent) } \\
& \text { s. } \mathrm{M}_{\mathrm{c}}(\mathrm{s} \cdot \mathrm{pcc})=\text { Call fid } \overline{\mathrm{e}} \quad \mathrm{s} \rightarrow \mathrm{s}^{\prime} \\
& \frac{\mathrm{s} \cdot \mathrm{pcc} \subseteq \operatorname{dom}\left(\mathrm{p} \cdot \mathrm{M}_{\mathrm{c}}\right) \quad \mathrm{s}^{\prime} \cdot \mathrm{pcc} \subseteq \operatorname{dom}\left(\mathrm{p} \cdot \mathrm{M}_{\mathrm{c}}\right)}{(\mathrm{s}, \varsigma) \stackrel{\tau}{{ }_{\mathrm{p}}}\left(\mathrm{s}^{\prime}, \varsigma\right)}
\end{aligned}
$$

Figure 2 gives two rules of the trace-step relation $\stackrel{\lambda}{p}_{p}$ : one where the label emitted is informative (namely, an input return label), and the other where the label emitted is not informative $(\tau)$. Notice that the assumptions of both Rules Return-to-program and Call-to-program-silent perform a check on the program counter capability pcc both before and after the execution step (i.e., in states $\mathrm{s}$ and $\mathrm{s}^{\prime}$ ). If this capability value is changed with respect to the program-ofinterest's code memory $\left(\mathrm{p} . \mathrm{M}_{\mathrm{c}}\right)$ by the execution step, then this indicates that control went into (or out of) the program of interest $\mathrm{p}$. We call such a change of control with respect to the program of interest a border-crossing action, and we give it an informative label that records the memory snapshot and that updates the auxiliary information (computing $\varsigma^{\prime}$ ). If on the other hand, the capability value in pcc is not changed (with respect to $\mathrm{p} . \mathrm{M}_{\mathrm{c}}$ ) by the execution step, then this indicates no interesting change of control took place. We call such an uninteresting action an internal action (i.e., internal to either the program $\mathrm{p}$ or to the context), and give it the uninformative label $(\tau)$. 
All uninformative labels $(\tau)$ are eventually dropped, and we concatenate only the informative labels into a trace prefix $\alpha$. These technicalities (all worked out in the supplementary material) are mostly inspired by process calculi [20]. Also, the idea of a trace label containing a shared-memory dump, and of the auxiliary information $\varsigma$ recording a summary of the shared addresses is inspired by Laird's [21] trace semantics for a lambda calculus with general references.

One key result of the choice of these ?- and !- decorated trace labels, is that the trace prefixes formed by the concatenation of the non-silent labels are alternating. By alternating, we mean that the decorators "?" for input and "!" for output occur alternately on the trace prefix. This is true of any trace prefix $\alpha$ in the set of traces $\operatorname{Tr}(p)$ of a program $p$ in both our target language CHERIExpress and the source one ImpMod:

Fact 1 (Traces are alternating). $\alpha \in \operatorname{Tr}(p) \Longrightarrow \alpha \in$ Alt $\checkmark^{*}$ where Alt $\stackrel{\text { def }}{=}(? \mid \epsilon)(! ?)^{*}(! \mid \epsilon)$ and $\dot{?}$ is the set of ?decorated labels, and similarly for $!^{5}{ }^{5}$

\section{Using TrICL simulation to prove that our PAC compiler preserves trace equivalence}

Having introduced how our auxiliary trace semantics works, we return back to Lemma 2, which states (in the contrapositive) that our compiler preserves trace equivalence. Our way to prove this lemma is to reduce it to the following two lemmas by unfolding Definition 2:

Lemma 4 (No trace is omitted by compilation). $\forall \alpha, p_{\mathrm{s}} . \alpha \in \operatorname{Tr}\left(\mathrm{p}_{\mathrm{s}}\right) \Longrightarrow \alpha \in \operatorname{Tr}\left(\llbracket \mathrm{p}_{\mathrm{s}} \rrbracket\right)$

Lemma 5 (No trace is added by compilation). $\forall \alpha, \mathrm{p}_{\mathrm{s}} . \alpha \in \operatorname{Tr}\left(\mathrm{p}_{\mathrm{s}}\right) \Longleftarrow \alpha \in \operatorname{Tr}\left(\llbracket \mathrm{p}_{\mathrm{s}} \rrbracket\right)$

The first (Lemma 4) follows easily by lifting the compiler forward-simulation result (which we prove about the execution steps) to trace steps. The second lemma (Lemma 5), however, is a bit involved, and requires extending the compiler forwardand backward- simulation relation into an alternating crosslanguage relation, namely $\operatorname{Tr} I C L$, that is indexed by an execution trace. The alternation of the $\operatorname{Tr} I C L$ simulation relation relies on Fact 1 about the alternation of traces.

To understand how the proof of Lemma 5 works, we first illustrate how an example trace prefix $\alpha$ emitted by an example compilation would look like. Example 4 portrays a trace prefix that is emitted by the compiled version of the example ImpMod module of Listing II.1 (with indications of each part of the action). For simplicity, we take our program of interest to be this singleton list of modules. In general, a program of interest can consist of many modules.

\footnotetext{
${ }^{5}$ The $*$ in $\checkmark^{*}$ is because a terminal state loops forever in our model.
}

Example 4 (Trace prefix of the compilation of the program in Listing II.1).

$$
\begin{aligned}
& \operatorname{call} \overbrace{(\text { send_rcv })}^{\text {fid }} \overbrace{[(\delta, \sigma, \sigma+512,0)]}^{\bar{v}} \text { ! } \\
& \underbrace{[\sigma \mapsto 0, \ldots, \sigma+42 \mapsto 4242, \ldots, \sigma+511 \mapsto 0]}_{\text {Mem }}, \underbrace{-1}_{\text {nalloc }} \\
& :: \text { ret } ? \overbrace{[\sigma \mapsto 0, \ldots, \sigma+42 \mapsto 0, \ldots, \sigma+511 \mapsto 0]}, \overbrace{-1}
\end{aligned}
$$

In the example, the compilation of the first three commands generates silent steps, which are dropped from traces and therefore not shown. Then the two non-silent labels illustrate the two cases of the proof of the alternating $\operatorname{Tr} I C L$ simulation:

1) the function call on Line 11 is border crossing, so its compilation emits a non-silent label, namely, an output call label which contains the callee function id (send_rcv), the argument to the call (the $\delta$-capability representing the pointer \&iobuffer), the direction of the call (! denoting output, i.e., program-to-context), a dump of the memory shared so far (namely, the contents of the array ioubuffer ), and the value -1 denoting the first heap address (the heap grows towards negative addresses).

2) the target context (in which our compiled program executes) returns control to the program after zeroing out the contents of the shared memory, emitting the input return label that is shown last in Example 4.

Notice that the goal from Lemma 5 is to show that exactly the given "target" trace prefix of Example 4 can be emitted by the source program (in some source context).

We show in Listing IV.1 a source context that (when linked with the program in Listing II.1) emits the trace in Example 4.

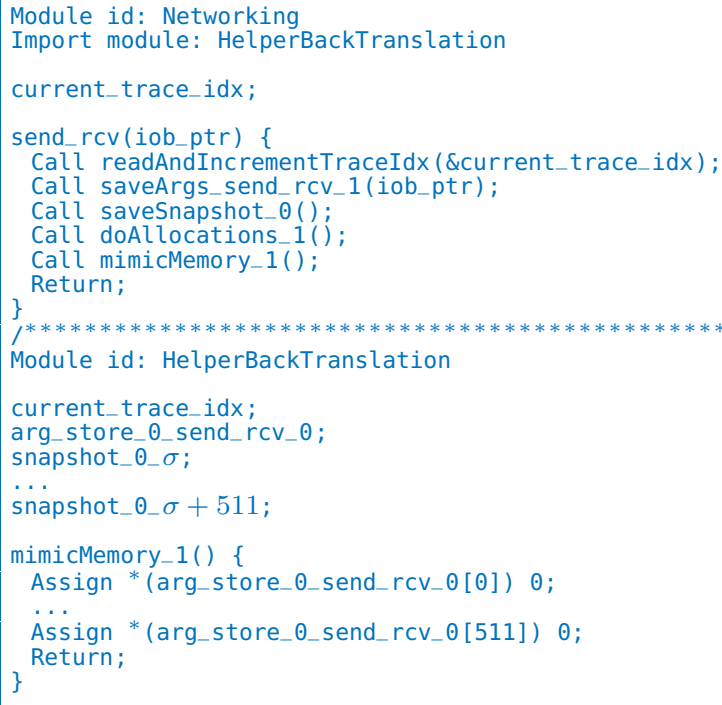

Listing IV.1: Example back-translation (simplified excerpt): An ImpMod context emulating the trace of Example 4.

The emulating context consists of two modules, Networking, which implements the API function send_rcv, and HelperBackTranslation, which implements helper 
functions and maintains metadata. We show just one example of such a helper function, namely mimicMemory_1(). mimicMemory_1() is called (on Line 11) by send_rcv(), so that the former zeroes out the IO buffer. The way the IO buffer is accessible at all by mimicMemory_1() is through the pointer stored in the global variable arg_store_0_send_rcv_0 (Line 18). This pointer would have been stored (not shown) by the function call saveArgs_send_rcv_1(iob_ptr); on Line 8.

We briefly explain what each helper function does. First, notice that given a finite trace prefix, the context that emulates this trace prefix defines a set of helper functions per trace label. The index of the corresponding trace label appears in the identifier of a helper function (for example, mimicMemory_1(), and saveSnapshot_0()).

To explain the helper functions, we follow the body of send_rcv(iob_ptr) line by line. In the beginning, the call to readAndIncrement Traceldx keeps track of the current position in the trace. This knowledge of the current position in the trace is not used in our toy example, but it would be used if the API function (send_rcv in this case) were called at more than one trace position; at each trace position, we would use this knowledge to call the corresponding helper functions (e.g., mimicMemory_3() instead of mimicMemory_1(), which would carbon copy to the shared memory the values that appear in trace position 3 instead of 1).

Next, on Line 8, we store the pointer iob_ptr in a global variable in the HelperBackTranslation module (as explained above). The reason we need to store it in a global variable is that we may need to use it in a future trace position, not just in the current call to send_rcv. For the same reason (the potential future use), we save (on Line 9) a snapshot of the whole shared memory in global variables snapshot_0_ $\sigma$ to snapshot_0_o +511 .

Next, on Lines 10 to 12 , the actual emulation of the trace action at trace position 1 is done. In our toy example, doAllocations_1() would do nothing because nalloc in Example 4 does not change. Then, mimicMemory_1() writes all the values (the zeros) to the shared memory before send_rcv eventually returns, transferring control from the emulating source context back to the program of interest.

The "vertical gap" challenge and how the $\operatorname{Tr} I C L$ relation solves it: Notice two main differences between the internal behavior of the emulating source context (that we explained above) and the behavior of the target context:

(i) the specific sequence of updates to the shared memory (before returning to the program of interest), and

(ii) the sequence of internal function calls (before returning to the program of interest)

These two differences mean that respectively the memory and the trusted call stack do not remain in sync (between the emulating source context and the given target context). We call this lack of sync between the execution states of the source and target contexts the vertical gap challenge.

The vertical gap is a challenge we face when attempting to define a cross-language relation between the given target execution and its emulating source execution. Notice that by defining the traces to capture only the observable behavior (and not the internal execution) of the program/context, we have created this vertical gap. However, the reason we defined the traces this way is we want to allow more freedom to what the back-translation function is rather than restricting this function to be the "inverse-compilation" function (because such a restriction would propagate assumptions that weaken our threat model, assumptions that would require a target context be only a context resulting from compilation).

Having acknowledged that the vertical gap is an inherent challenge of our threat model and of the nature of our languages (the languages that, unlike prior work $[16,17$, $18,22,23]$, allow fine-grained memory sharing), we designed the $\operatorname{Tr} I C L$ relation, which solves the vertical gap by relying on an alternating strong/weak similarity, and by introducing a mediator execution.

The $\operatorname{Tr} I C L$ relation is defined in terms of strong $(\approx)$ and weak $(\sim)$ similarities, and in terms of the relation for whole-program compiler-correctness $\left(\cong_{\mathrm{p}}\right)$. That $\operatorname{Tr} I C L$ reuses the compiler-correctness relation $\left(\cong_{p}\right)$ is one of the main advantages of $\operatorname{Tr} I C L$.

Definition 3 (Trace-Indexed Cross-Language ( $\operatorname{Tr} I C L)$ alternating simulation relation).

$$
\begin{aligned}
& \operatorname{Tr} \operatorname{ICL}\left(\mathrm{s}_{\mathrm{emu}}, \mathrm{s}_{\mathrm{med}}, \mathrm{S}_{\text {given }}, \varsigma\right)_{\alpha, i, \mathrm{p}} \stackrel{\text { def }}{=} \\
& \mathrm{s}_{\mathrm{emu}} \cong \mathrm{s}_{\mathrm{med}} \\
& \wedge \text { emulate_invariants }\left(\mathrm{s}_{\mathrm{emu}}\right)_{\alpha, i, \mathrm{p}} \\
& \wedge \alpha(i) \in \dot{i} \Longrightarrow\left(\mathrm{s}_{\text {med }}, \varsigma\right) \approx_{\llbracket \mathrm{p} \rrbracket}\left(\mathrm{s}_{\text {given }}, \varsigma\right) \\
& \wedge \alpha(i) \in \dot{?} \Longrightarrow\left(\mathrm{s}_{\text {med }}, \varsigma\right) \sim_{\llbracket \mathrm{p} \rrbracket}\left(\mathrm{s}_{\text {given }}, \varsigma\right)
\end{aligned}
$$

The $\operatorname{Tr} I C L$ relation helps us prove Lemma 5 because we can rely on the fact that $\operatorname{Tr} I C L$ satisfies the following alternating backward-simulation condition:

Lemma 6 ( $\operatorname{Tr} I C L$ step-wise alternating backward-simulation).

$$
\begin{aligned}
& \alpha \in \mathrm{Alt} \wedge \\
& \operatorname{Tr} \operatorname{ICL}\left(\mathrm{s}_{\mathrm{emu}}, \mathrm{s}_{\mathrm{med}}, \mathrm{S}_{\text {given }}, \varsigma\right)_{\alpha, i, \mathrm{p}} \wedge \\
& \left(\text { Sgiven }_{\text {give }} \varsigma\right) \stackrel{\alpha(i)}{\longrightarrow \mathrm{p} \rrbracket}\left(\mathrm{s}_{\text {given }}^{\prime}, \varsigma^{\prime}\right) \\
& \Longrightarrow \\
& \exists \mathrm{s}_{\mathrm{emu}}^{\prime}, \mathrm{s}_{\mathrm{med}}^{\prime} \text {. } \\
& \left(\mathrm{s}_{\mathrm{emu}}, \varsigma\right) \stackrel{\alpha(i)}{\mathrm{p}_{\mathrm{p}}}\left(\mathrm{s}_{\mathrm{emu}}^{\prime}, \varsigma^{\prime}\right) \wedge
\end{aligned}
$$

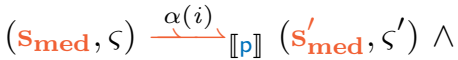

$$
\begin{aligned}
& \operatorname{Tr} \operatorname{ICL}\left(\mathrm{s}_{\mathrm{emu}}^{\prime}, \mathrm{s}_{\text {med }}^{\prime}, \mathrm{s}_{\text {given }}^{\prime}, \varsigma^{\prime}\right)_{\alpha, i+1, \mathrm{p}}
\end{aligned}
$$

Observe that the $\operatorname{Tr} I C L$ relation is a ternary ${ }^{6}$ relation between two target states and a source state:

\footnotetext{
${ }^{6}$ The auxiliary information $\varsigma$ is considered part of the three trace-states, hence we do not count it in the arity, and only count the three trace-states.
} 
1) the source state, which comes from the execution of $\mathfrak{C}_{\mathrm{emu}}[\mathrm{p}]$, which is the program of interest $\mathrm{p}$ linked with the emulating source context $\mathfrak{C}_{\text {emu }}$ (Listing IV.1 is an example $\mathfrak{C}_{\mathrm{emu}}$ ),

2) the first target state, namely, the mediator state, which comes from the execution of $\llbracket \mathfrak{C}_{\mathrm{emu}} \rrbracket[\llbracket p \rrbracket]$, which is the translation of both the program of interest and the emulating context, and

3 ) the second target state, namely, the given state, which comes from the execution of $\mathfrak{C}_{\text {given }}[\llbracket \mathrm{p} \rrbracket]$, which is the translation of the program of interest, linked with a target context.

$\operatorname{Tr} I C L$ introduces a "mediator" execution (state $\mathrm{s}_{\text {med }}$ ) between the execution of the back-translated source context (state $\mathrm{S}_{\mathrm{emu}}$ ), and the execution of the given target context (state Sgiven). This mediator execution enables us to conveniently re-use both compiler backward- and forward- simulations. (In Definition 3 above, notice that independently of the decorator/parity of the label $\alpha(i)$, we need in Lemma 6 to re-establish the compiler correctness relation $\left(\cong_{p}\right)$. There is where we in one case need compiler backward-simulation and in the other forward-simulation).

$\operatorname{Tr} I C L$ helped us solve the vertical gap because it reduced the lack of sync problem between $\mathrm{S}_{\mathrm{emu}}$ and $\mathrm{s}_{\text {given }}$ to a lack of sync between $\mathrm{s}_{\text {med }}$ and sgiven. The latter is easier because it can be captured using a same-language invariant, namely the strong/weak similarity relation. The key idea of the strong/weak similarity is to characterize the strong relation $\left(\approx_{\llbracket \mathrm{p} \rrbracket}\right)$ by equality of the whole reachable memory, and the weak relation $\left(\sim_{\llbracket p \rrbracket}\right)$ by equality of the unreachable memory, i.e., the memory that is private to the program of interest $\llbracket p \rrbracket$. Because the memory private to $\llbracket p \rrbracket$ is by definition untouchable by the emulating context, weak similarity is weak enough to allow the (compiled) emulating context to do an arbitrary sequence of memory updates (and internal function calls). Weak similarity is however just strong enough to be restrengthened upon a successful emulation of the input (?) step. (Notice from Definition 3 that weak similarity holds when the context is executing-identified by the next label $\alpha(i)$ being an input (?) to the program of interest).

Due to lack of space, we defer lots of proof details about the strong and weak similarity relations to the technical report. Most interestingly, we introduce there the idea of a successorpreserving isomorphism and use it to define the stack similarity relations that allow the internal function calls to differ between the stack of $\mathrm{s}_{\text {med }}$ and the stack of sgiven.

We focused in Section IV-D on the proof of Lemma 2 because we think it is the most interesting (compared to Lemmas 1 and 3). Nevertheless, it is worth mentioning that for the proof of Lemma 1, we actually reuse the idea of the strong and weak similarity relations that we just introduced above. Also, for Lemma 3, we use a construction similar to the back-translation that we introduced in Listing IV.1.

\section{IMPLEMENTING AUTOMATIC MODULE-BASED} ISOLATION FOR C USING THE CHERI INFRASTRUCTURE

The $C H E R I$ compiler already implements the $P A C$ principle, but it does not yet implement a module-based isolation scheme like our ImpMod does. This section presents how to implement such a scheme as a source-to-source $\mathrm{C}$ compiler that annotates $\mathrm{C}$ programs with compartmentalization directives that, in turn, the CHERI system (including the CHERI Clang/LLVM) [4] understands and implements. This provides a way to automatically enforce our module-based isolation for real-world $C$ programs. The implementation of this sourceto-source compiler relied on extensions to libcheri that we detail in Section V-C.

\section{A. Zibcheri}

In order to present the source-to-source compiler, we first briefly explain libcheri [24], CheriBSD's programmerfriendly interface to the CHERI compartmentalization features. Under libcheri, the isolated, compartmentalized parts of a program are called sandboxes and libcheri is the API for loading and invoking sandboxes.

CHERI's in-process sandboxes are objects which are represented in CHERI assembly using invokable object capabilities [24]. The creation of these objects is the responsibility of the programmer who groups functions into classes and creates an invokable object capability of the class type. The programmer does that by annotating function declarations as cheri_ccall so that conventional function calls are replaced by object capability invocations. Functions that are exported by the current sandbox should be annotated with the cheri_ccallee attribute. At run time, libcheri loads the executable images from the file system and creates the respective object capabilities. It is critical that, when the sandbox loading routines are called, the program is in its initial state where it has control over its entire address space as well as file system access.

Finally, constructor functions sandboxes_init must be added to the modules that have call sites to external functions. These constructor functions essentially perform a second linking phase to correctly initialize the references that modules hold to each other's exported functions.

Listing V.1 below shows how these annotations are added to (a subset of) the code snippet of Listing II.1.

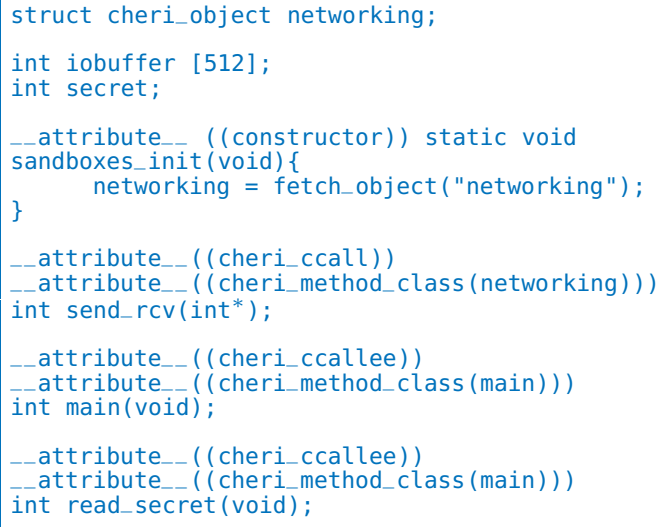

Listing V.1: Adding CHERI annotations to existing code. 


\section{B. Source-to-source transformations}

Our compilation scheme maps each $\mathrm{C}$ module to a separate sandbox, i.e., it assigns a CHERI class and creates a CHERI object for each module. To do this, our compiler creates a mapping from function identifiers to $\mathrm{C}$ modules, which helps resolve dependencies in the next step. Then it traverses each module's AST and annotates every external function declaration it encounters as either cheri_ccall or cheri_ccallee, depending on whether the function is defined in the current translation unit or is exported (more examples are available in the Appendix Listings A.1 to A.4). As a performance optimization over the ImpMod model, intra-module function calls do not translate to object capability invocations. Instead, they are ordinary MIPS function calls. This change has no security implications since the module is the unit of trust.

The initialization of sandboxes required some extensions to libcheri, which we now describe.

\section{Libcheri extensions}

One hurdle in using libcheri for our compilation scheme is initialization. libcheri exports two functions for sandbox creation: sandbox_class_new, which instantiates the class, and sandbox_object_new, which creates an object of a certain class. As stated in Section V-A, the calls to these two functions need to take place when the program is at its initial state with control over the entire address space. We implement this by annotating the initialization functions for all of the potentially required sandboxes as constructor functions, which are invoked at the very beginning before executing the main function.

Concretely, we extend libcheri with a new load/initialization function, sandbox_chain_load(). This function is meant to be called only once by an initialization module, which is the only privileged part of the program (and hence can invoke system calls and create sandboxes). The initialization function sandbox_chain_load() loads the main sandbox, the module implementing the main() function, from the file system and also any modules that main depends on (recursively). It also creates relevant object capabilities for every sandbox and places them at the beginning of the sandbox's data segment. As a result, every sandbox has access to the object capabilities necessary to invoke exported functions from other sandboxes. Extending libcheri required considerable additions to the libcheri code base including the definition of sandbox_chain_load(), new versions of sandbox creation routines that support sandbox dependencies and low-level macros that expose relevant sandbox metadata to $\mathrm{C}$.

\section{EXPERIMENTAL EVALUATION}

Evaluating our module-based isolation scheme and its proofof-concept implementation is a matter of application support and performance. In terms of support, it is important to establish the viability of building and executing real-world $\mathrm{C}$ applications under our scheme (Section VI-A) while performance measurements are necessary in order to reveal the overhead that our scheme introduces (Section VI-B).

\section{A. Manual code changes}

We ported four large open-source $\mathrm{C}$ libraries, and carefully chose a heterogeneous set of examples: zlib [25], LibYAML [26], GNU-barcode [27], and libpng [28]. These programs can be built using CHERI's $P A C$ compiler, which only translates pointers to capabilities, but the situation is trickier after we apply our source-to-source transformations. In particular, there are additional constraints in $\mathrm{C}$ code when using sandboxes in CHERI and there is no guarantee that the code will adhere to them and as a result, we encountered a number of incompatible programming patterns.

TABLE I: Incompatible programming patterns

\begin{tabular}{|l|l|l|l|l|}
\hline Software & zlib & LibYAML & GNU-barcode & libpng \\
\hline Passed local ref & 2 & 15 & 0 & 13 \\
\hline Extern global var & 3 & 0 & 0 & 0 \\
\hline Pointer to ext fun & 2 & 1 & 26 & 2 \\
\hline Other & 0 & 1 & 1 & 4 \\
\hline Total & 7 & 17 & 27 & 19 \\
\hline
\end{tabular}

TABLE II: Porting overhead

\begin{tabular}{|l|l|l|l|l|}
\hline Software & zlib & LibYAML & GNU-barcode & libpng \\
\hline Lines of code & 11255 & 12762 & 4657 & 33029 \\
\hline Altered lines & 130 & 114 & 164 & 51 \\
\hline Percentage & 1.15 & 0.89 & 3.5 & 0.15 \\
\hline
\end{tabular}

Table I summarizes the frequency of occurrence of each type of incompatible pattern that we found in each of these example libraries. Table II summarizes the amount of code change $^{7}$ that we needed to make (manually) so that we can compile and successfully run the benchmarks for each of these example libraries. Overall, Tables I and II let us conclude that the amount of code changes we had to make is rather small. We discuss the three most common, and interesting, incompatible patterns (in row order).

a) Pointers to local variables passed as argument to an external function: CHERI prohibits passing local capabilities when invoking object capabilities and this pattern will trigger the violation. The solution is simple as a semantically equivalent result can be obtained by moving the guilty variables to the heap, either as a global variable or dynamically allocated. This is a fundamental limitation of CHERI and is reflected in our theoretical model. More on the issue of temporal safety on capability machines can be found in recent work $[6,7,8]$.

b) External global variables: Our compiler supports global variables that are used in the same translation unit they are defined in, regardless of their qualifiers. It does not, however, support the declaration or usage of a global variable that is defined in a different translation unit. Instead, the importing translation unit may use a pointer to that variable,

\footnotetext{
${ }^{7}$ Note that the measurements come from the UNIX utility diff, where modifying a line counts as two lines modified: one for deleting the original and one for adding its replacement. Having said that, the ratio was very low with zlib, LibYAML and libpng at a remarkable $1.15 \%$ and $0.89 \%$ and $0.15 \%$ respectively with GNU - barcode at a slightly higher 3.5\%
} 
where this pointer is explicitly imported at run time (ideally during an initialization phase by calling a getter function). Full support would require additional work to the $C H E R I$ linker.

c) External function pointers: In CHERI, a C function pointer is always compiled to an executable capability. This can be problematic under our scheme in the case where the pointee function belongs to a different module. In the easy case where we can determine the pointee statically, we can do away with the function pointer altogether and replace it with the pointee. If on the other hand, the decision of which callback function to provide is made by the provider only at runtime, then a major re-factoring is needed. This re-factoring modifies both the callback provider and the callback consumers. If the callback consumer is however an arbitrary client library, then our source-to-source transformation alone will not be able to handle this without forcing the programmer to rewrite the API (giving up function pointers) and consequently to change every client library (i.e., every use of the API).

\section{B. Performance}

To measure the performance of our system, we carried out benchmarks on the software we ported to our scheme. We compared the secure, compartmentalized version of ported software with one running in a single sandbox. This is because unsandboxed execution has a significantly altered executing environment w.r.t when sandboxes are involved, and that heavily affects performance. The memory allocator is a telling example, as the one used for sandboxed execution is less memory efficient but much faster. So a single-sandbox execution environment "evens up" the playing field yielding measurements of the actual overhead. The benchmarks were performed on a $C H E R I$ virtual machine (implementing CHERI ISA version 5 [29]) running our modified version of CheriBSD.

Before presenting the results, it is important to briefly discuss the main factor that affects performance, namely object capability invocation. As mentioned in Section V, our compiler only affects declarations of external functions while $\mathrm{C}$ statements remain unaltered. Consequently the transformed executable should differ from the original only on the invocation method of external functions. The rest of the instructions, representing the parts of the compiled program that are not related to object invocation, are identical.

The performance overhead of object capability invocation has been covered in earlier literature [4], measuring at about 500 machine cycles per invocation. This cost includes userspace clearing of unused argument registers, saving and restoring registers and kernel-space argument validation. Various optimization techniques are being explored [3] and partially implemented, as the latest published version of the $C H E R I$ specification [11] supports non-exception-based domain transitions. However, the performance gains are either speculative [3] or otherwise unexplored.

What this means is that performance boils down to the number of external function calls in relation to the rest of the program's statements: A higher proportional number of external function calls would translate to a higher number of object capability invocations and a more pronounced delay. This is, of course, a characteristic of individual software and not of our compiler, thus begging the question of whether or not the number of external function calls in real-world software is sparse enough to guarantee an acceptable performance overhead when compiling it under our scheme.

The benchmark results are summarized in Figure 3. Note that GNU-barcode is designed to process bar codes of limited size so there was no point in running benchmarks for it. Each of the three remaining software demonstrate a distinct case of overhead while all of them confirm that the number of sandbox invocations is by far the biggest delay factor. There is also a small, constant delay of about 0.2 seconds associated with the sandbox loading routines.

The first benchmark was that of zlib found in Figure $3 a$. The overall number of sandbox invocations was very low throughout, irrespective of the size of the input data, ranging from 43 to 441 . This is reflected in the figure as the delay is consistently minor.

The case of LibYAML proved very different. As it turns out, the number of sandbox invocations were increasing in linear fashion to the size of the input data. Although initially the sandbox invocations were relatively low at 1482, they reached up to 1523546 . This is reflected in Figure $3 \mathrm{~b}$ where the execution time difference between the two versions increases with each iteration, while the relative delay is consistently between $35 \%$ and $40 \%$.

The final case, libpng, was that of a program with a relatively high number of sandbox invocations (about 125k) that remains more or less constant irrespective of the input data. This translates to a perceptible, constant delay throughout, as one can see in Figure 3c.

Our benchmarks confirm that the number of sandbox invocations is the most significant overhead factor. Moreover, each sandbox invocation introduces a constant amount of delay (see Figure 4). In this figure, the $x$-axis is the number of sandbox invocations in logarithmic scale and the $y$-axis is in seconds. We plot the absolute execution time difference between the two versions for each of the three test cases. For zlib, the plot is an almost flat line as the number of sandbox invocations increases very slightly. The plot for libpng is basically a point as neither the sandbox invocations or the delay changes in any perceptible way. The most interesting graph is that for LibYAML, where the delay increases in a perfect, linear manner relative to the increase of the sandbox invocations (it is not easy to see as the $x$-axis is logarithmic).

As stated earlier, the overhead being acceptable or not for a particular piece of software boils down to the number of external function calls (which are eventually compiled to sandbox invocations) relative to the rest of the code. In general, we cannot make any assumptions on that metric for existing $\mathrm{C}$ software, so the question of viability of a certain piece of software for our scheme can only be answered on a per-case basis. 
(a) zlib

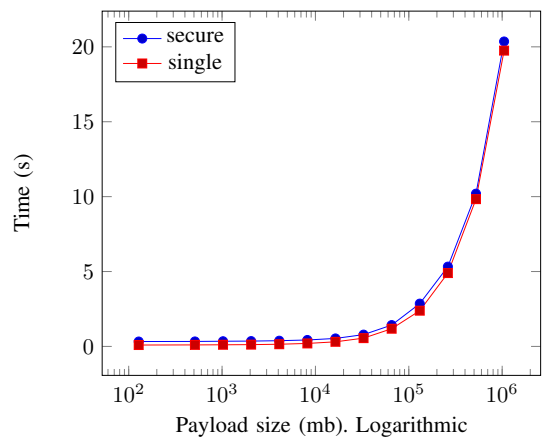

(b) LibYAML

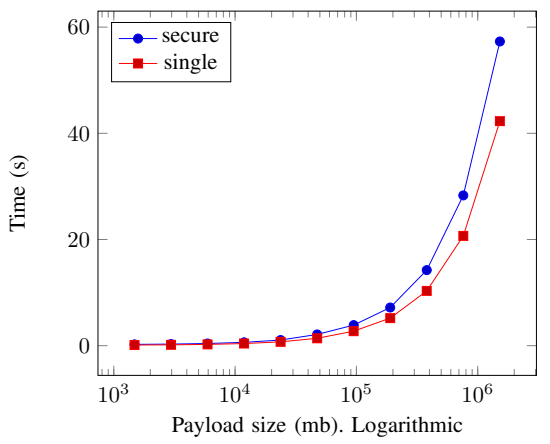

(c) libpng

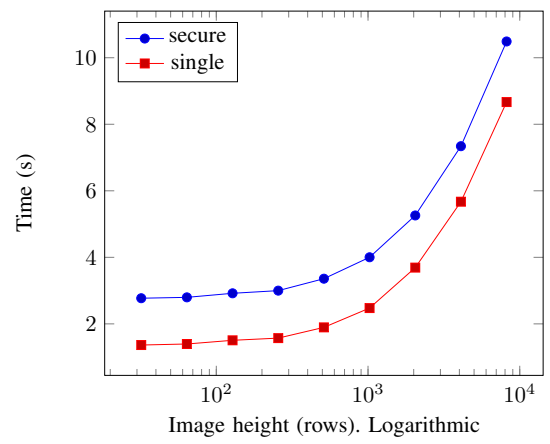

Fig. 3: Benchmark results for zlib, LibYAML and libpng respectively. Blue lines represent the secure, compartmentalized versions while red lines represent a single-sandbox execution. All $\mathrm{x}$ axes are in Log scale.

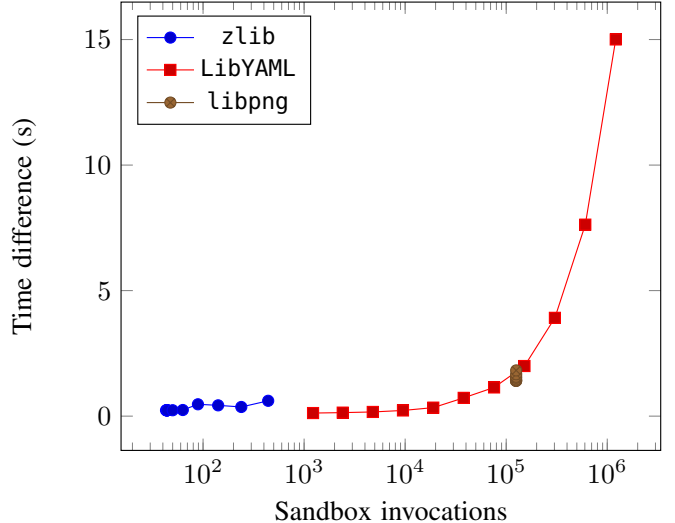

Fig. 4: Execution time difference relative to the number of sandbox invocations.

\section{LIMITATIONS OF THE MODEL AND FUTURE WORK}

While we believe our work is a significant step forward in the understanding of the security properties of $P A C$ compilers, it still makes some simplifications and assumptions that would be interesting to remove in future work.

Treatment of memory management: Memory allocation in our model is oversimplified and does not support de-allocation. This simplification allows us to represent the state of the memory allocator as just the next-free-address, and this is essential in keeping our model manageable. To the best of our knowledge, nobody has yet developed fully abstract trace semantics for languages with a realistic model of deallocation. In practice, this means that our formal results do not rule out that compiled programs become distinguishable (i.e. leak information) through the way they manage memory.

Other side-channels: Programs can leak information to their context through a whole variety of other softwareexploitable side-channels. A context can measure execution time of a program, or determine the memory locations that the program has accessed through cache-based side-channels [30]. We do not model these channels. As a consequence, our compiler also is not guaranteed to preserve resistance against such attacks [31]. There is however recent related work that specifically investigates how to secure compilers such that they preserve side-channel resistance [32, 33, 34].

Possibly redundant load-time checks: Our compiler relies on the linker/loader to perform some checks on machine code before it can be linked. For instance, to avoid confusion between data and code capabilities, the loader should check the linked code does not access pcc directly. (In our formalization, this is modeled by the absence of a getppc primitive.) While some of these checks (like the one above) are justifiable from a security perspective, some are just artifacts of the proof strategy [35], and do not seem essential for security. One example is that we impose that the context's data segment must be in memory after the program's data segment. There is no security motivation for this check; it just makes the proof easier: the construction of the back-translated context will occupy a data segment whose size is in principle larger (due to meta-data) than the size of the data segment of the target context that we are back-translating. This check ensures that this increase in size does not impact the position of the program's variables in memory. A more complex proof (or a different proof technique) might remove the need for such checks.

\section{CONCLUSION AND RELATED WORK}

We presented a proof of full abstraction of the compiler ImpMod to CHERIExpress which we called a "pointersas-capabilities" compiler. This compiler that we model should be understood as a specification for compilers that target architectures like CHERI. In addition, we have provided evidence for the feasibility of implementing this specification by reporting on a proof-of-concept implementation.

Our paper is the first to formally show that $P A C$ compilers satisfy a strong full abstraction property. But there is a rich body of related work studying security proofs for compilers, and compilation to capability machines. We briefly summarize related work and the relation to our results. 


\section{A. Compilation to capability machines}

The closest work to ours is StkTokens by Skorstengaard et al. [8]. They also model and verify a compiler transformation in a compiler that targets capability architectures. But there are broadly two differences to highlight between their model and ours. First, they model and verify a compiler transformation that implements a calling convention, rather than a $P A C$ transformation. Second, their compiler targets a linear capability machine, meaning one that supports a special form of capabilities which is subject to a certain non-re-usability check that they assume is supported in hardware. Our model does not assume this particular hardware support.

The calling convention that they implement relies carefully on linear capabilities. Instead of CHERIExpress's stackper-component design (for data stacks), the use of linear capabilities enables sharing the same unified control and data stack among all components including mutually distrustful ones: the (untrusted) callee is handed a linear capability on the stack. Then after control returns, by convention, the caller checks for the existence of the same capability, thereby detecting any (malicious or buggy) retention of the stack access permission. In contrast, in CHERIExpress which lacks linear capabilities, a trusted control stack is assumed to be maintained in kernel space, thereby eliminating even the need to hand out a capability on this stack to any userspace program - hence, no retention of such a stack capability by a malicious component is at all possible. For storing the data part of the call frames, every component is allocated its own data stack. The assumption that the kernel is responsible for the control stack is compatible with the current CHERI ISA [11] specification. ${ }^{8}$

\section{B. Trace equivalence}

Laird [21] gives a fully-abstract trace semantics for a functional language with general references. Our trace labels for CHERIExpress are inspired by this work. Laird relies on a bipartite LTS in which nodes are partitioned between programconfigurations and environment-configurations (we used the word "context" instead of environment). We, however, do not build into CHERIExpress this explicit segregation between program and environment configurations. Instead, we perform a simple check on the status of the capability registers before and after a step in order to decide the direction of a trace action. Relying on this check, Fact 1 ensures that programs interact with the context in precisely the desired alternating fashion that a bipartition would have ensured.

The fact that we chose to use trace equivalence at all (to characterize contextual equivalence) is inspired by existing work that use it in proofs of full abstraction of compilation to Protected Module Architectures (PMAs) and tagged architectures $[16,17,18,22,36]$.

\footnotetext{
${ }^{8}$ The current CHERI ISA also includes an experimental design with support for linear capabilities.
}

\section{FA Proofs that do not use trace equivalence}

Back-translating traces to enable an easy cross-language simulation relation is not the only strategy that has been used in proofs of fully-abstract compilation (surveyed by Patrignani et al. [13]). Several other proof ideas exist:

- While Fournet et al. [37] also use labeled transitions, their full abstraction proof of the translation of an ML-like language to Javascript is based on bisimulation. We, on the other hand, require our TrICL (Trace-Indexed CrossLanguage) relation to only satisfy the coarser simulation condition.

- In their StkTokens work, Skorstengaard et al. [8] use another piece of machinery that does not require labeled transitions at all: a logical relation with recursive worlds.

- New et al. [38] rely on a shallow embedding of the target language (a $\lambda$-calculus with exceptions) into the source (a $\lambda$-calculus with recursive types) at a dynamic type, and use a cross-language relation that defines in multilanguage terms the contextual equivalence relation.

- Devriese et al. [39] coin the term approximate backtranslation to express the idea that the context that the back-translation produces only needs to behave similarly to the original (and unknown) context for a limited number of steps, namely, the number of steps in which it (the original context) terminates. They use step indexing to represent in the cross-language logical relation this approximate behavior.

When the target language is a subset of the source, as in the work by Crary [40] on fully-abstract module compilation, then full abstraction of the translation is a corollary of contextual equivalence between the input term and the output term of the translation.

\section{Other secure compilation criteria}

Abadi [10] was the first to promote full abstraction as a criterion for compiler-enforced protection. Abadi motivated the usefulness of full abstraction as a tool for understanding the design of secure implementations of programming languages by giving examples of security violations introduced by otherwise-functionally-correct implementations, and then explaining how those insecure implementations are not fully abstract.

Other criteria for telling the security of a compilation scheme involve proving what class of hyperproperties a compiler can preserve robustly, i.e., when its code is linked against arbitrary target code, as we do [23, 41, 42, 43]. For example, if a compiler needs to preserve noninterference-like properties, it suffices to prove that it preserves hypersafety properties (noninterference being in the class of hypersafety). Or, if a compiler needs to preserve integrity of compiled code, it suffices to prove that it preserves safety properties (integrity being in the class of safety). Unlike $F A$ most of these criteria require reasoning about the trace behaviour of a partial program. Since we have devised traces for our languages, we believe we can explore which of these criteria is applicable to our work as well. 
Another active line of work [32, 33, 34] is concerned with making sure that the compiler does not undo countermeasures that the programmer of cryptographic libraries implements in order to ensure protection against timing attacks or other secret-revealing attacks. In this line of work, the guarantees that are sufficient to capture the desired security intuition are believed to be weaker but more domain-specific than the full abstraction guarantee.

\section{APPENDIX}

\section{A. Semantics of ImpMod expressions}

In Figure 5, notice that rules Eval-arr-offset and Eval-var are just de-sugaring rules. Also, notice that for simplicity, expressions in ImpMod do not have side effects (the evaluation context $\mathrm{Fd}$, MVar, $\beta, \Delta, \Sigma$, Mem, $\Phi, \mathrm{pc}$ that we introduce shortly is actually read-only.).

The evaluation context for expressions consists of:

1) syntactic information about the program given by the function definitions $\mathrm{Fd}$, the declarations of module-global variables MVar, and the layout and bounds $\beta$ of all the program's variables;

2) load-time information about the program given by the per-module data-segment-location $\Delta$, and the per-module local-stack-location $\Sigma$; and

3) execution-state information given by the memory Mem, the stack pointers $\Phi$ of the module-local stacks and the program counter $\mathrm{pc}$.

\section{B. The TrICL proof technique}

The $\operatorname{Tr} I C L$ relation is indexed by the given trace $(\alpha)$ from Lemma 5 , and by a position $i$ on this trace.

The goal is to prove that the $\operatorname{TrICL}$ relation satisfies the step-wise backward simulation condition (Lemma 6). But first, we recall that $\operatorname{TrICL}$ is defined in terms of four main relations/invariants:

1) the vanilla (whole-program) compiler-correctness relation $\left(\cong_{p}\right)$ between the source state and the mediator state satisfying lifted forward- and backward-simulations (Lemmas 7 and 8)

2) a strong-similarity relation $\left(\approx_{\llbracket p \rrbracket}\right)$ between the mediator state and the given state, a relation that satisfies lock-step simulation (Lemma 9)

3) a weak-similarity relation $\left(\sim_{\llbracket p \rrbracket}\right)$ also between the mediator state and the given state, a relation that satisfies option simulation (Lemma 11), and together with the strong similarity satisfying both weakening (Lemma 10) and strengthening (Lemma 12)

4) emulation invariants about the source state satisfying both adequacy (Lemma 13) and preservation by trace steps (Lemma 14)

Notation: We write $s \stackrel{\alpha}{\rightarrow} s^{\prime}$ to denote that $s$ is a state of the program-of-interest $\mathrm{p}$ (linked with some context), and that $\alpha$ is a compressed trace prefix (i.e., with uninformative $(\tau)$ labels dropped) that is emitted by the (multiple-step) execution of $\mathrm{s}$ until $\mathrm{s}^{\prime}$. We write $\mathrm{s} \stackrel{\alpha}{\rightarrow} \mathrm{p} \mathrm{s}^{\prime}$ to denote the same about the target language.

Lemma 7 (Compiler forward-simulation lifted to compressed trace steps).

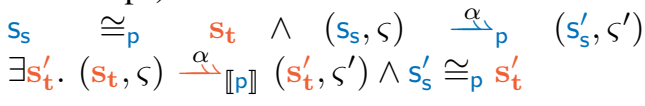

Lemma 8 (Compiler backward-simulation lifted to compressed trace steps). 
Fig. 5: Evaluation of expressions e in ImpMod. The evaluation relation $\Downarrow \subseteq$ e $\times \bigvee$ is defined on pairs of expressions e and values $\mathrm{V}$. The evaluation relation $\Downarrow$ is indexed with an evaluation context $\mathrm{Fd}$, MVar, $\beta, \Delta, \Sigma$, Mem, $\Phi$, pc. The evaluation context is used in rules Eval-amp-local-var, Eval-amp-module-var and Eval-star. Instead of writing Fd, MVar, $\beta, \Delta, \Sigma, \mathrm{Mem}, \Phi, \mathrm{pc} \vdash \mathrm{e} \Downarrow \mathrm{v}$, we abbreviate it as $e \Downarrow v$.

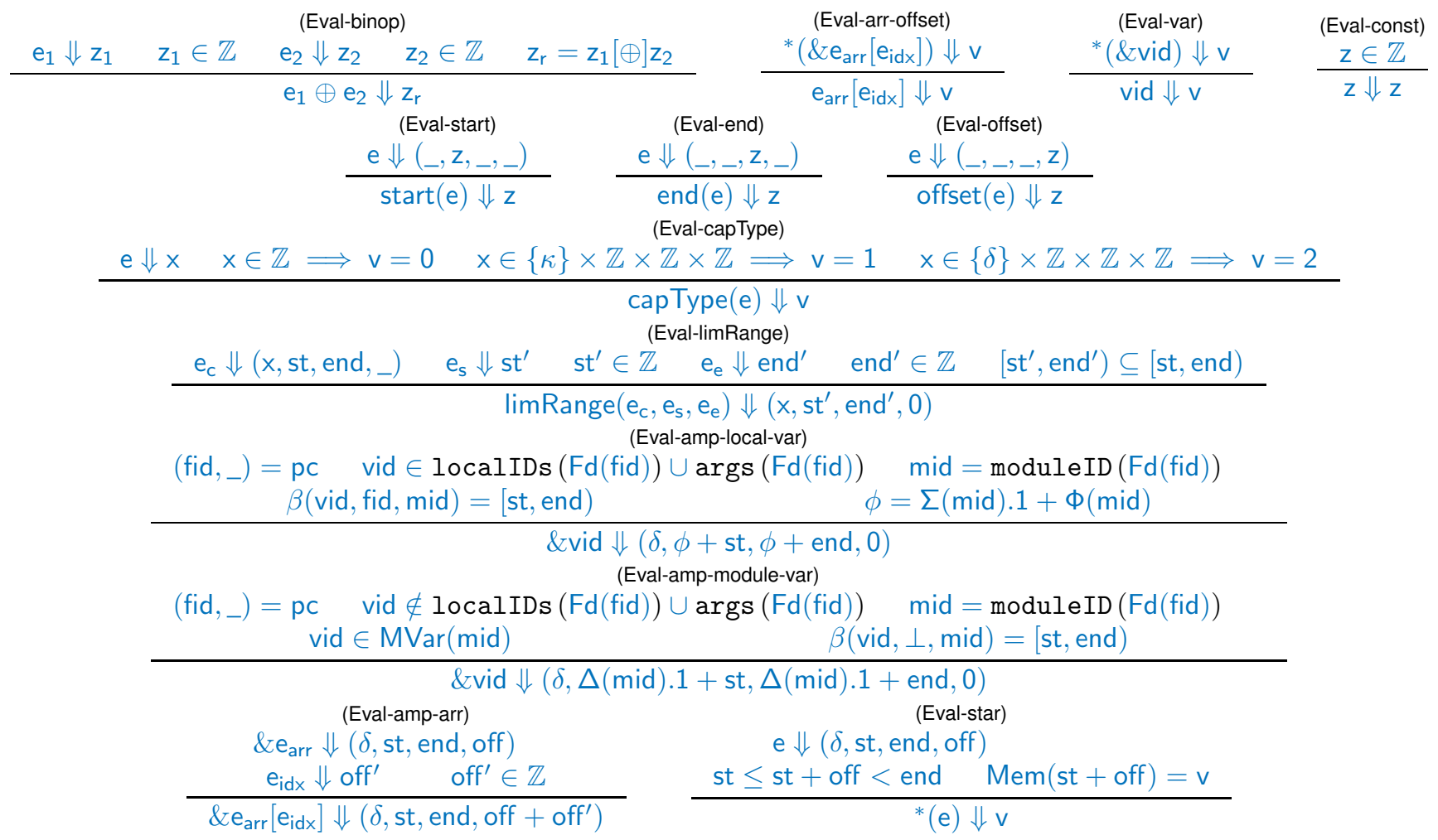

Fig. 6: (Excerpt) Small-step semantics of commands $C m d$ in ImpMod. The small-step relation $\rightarrow \subseteq s \times s$ is defined on pairs of execution states s. The small-step relation $\rightarrow$ is indexed with an evaluation context $\mathrm{Fd}, \mathrm{MVar}, \beta, \Delta, \Sigma$ and an allocation limit $\nabla$. The allocation limit $\nabla$ is used in rule Allocate.

$$
\begin{aligned}
& \text { (Assign-to-var-or-arr) } \\
& (\text { fid }, \mathrm{n})=\mathrm{pc} \quad \operatorname{commands}(\mathrm{Fd}(\mathrm{fid}))(\mathrm{n})=\text { Assign } \mathrm{e}_{\mathrm{I}} \mathrm{e}_{\mathrm{r}} \quad \operatorname{modID}=\operatorname{moduleID}(\mathrm{Fd}(\mathrm{fid})) \\
& \mathrm{Fd} \text {, MVar, } \beta, \Delta, \Sigma \text {, Mem, } \Phi, \mathrm{pc} \vdash \mathrm{e}_{\mathrm{l}} \Downarrow(\delta \text {, st, end, off }) \quad \mathrm{Fd}, \mathrm{MVar}, \beta, \Delta, \Sigma, \text { Mem, } \Phi, \mathrm{pc} \vdash \mathrm{e}_{\mathrm{r}} \Downarrow \mathrm{v} \\
& \forall \mathrm{st}^{\prime}, \text { end }^{\prime} . \mathrm{v}=\left(\delta, \mathrm{st}^{\prime}, \mathrm{end}^{\prime}{ }_{-}\right) \Longrightarrow\left(\left[\mathrm{st}^{\prime}, \mathrm{end}^{\prime}\right) \cap \Sigma(\operatorname{modID})=\emptyset \vee[\text { st, end }) \subseteq \Sigma(\bmod I D)\right) \\
& \text { st } \leq \text { st }+ \text { off }<\text { end } \quad \operatorname{Mem}^{\prime}=\operatorname{Mem}[\text { st }+ \text { off } \mapsto v] \\
& \mathrm{Fd}, \mathrm{MVar}, \beta, \Delta, \Sigma, \nabla \vdash\langle\mathrm{Mem}, \Phi, \mathrm{pc} \text {, stk, nalloc }\rangle \rightarrow\left\langle\mathrm{Mem}^{\prime}, \Phi \text {, inc (pc), stk, nalloc }\right\rangle \\
& \text { (Allocate) } \\
& (\text { fid }, \mathrm{n})=p c \quad \text { commands }(\mathrm{Fd}(\mathrm{fid}))(\mathrm{n})=\text { Alloc e } \mathrm{e}_{\mathrm{size}} \\
& \mathrm{Fd}, \mathrm{MVar}, \beta, \Delta, \Sigma \text {, Mem, } \Phi, \mathrm{pc} \vdash \mathrm{e}_{\mathrm{I}} \Downarrow(\delta \text {, st, end, off }) \quad \mathrm{Fd}, \mathrm{MVar}, \beta, \Delta, \Sigma \text {, Mem, } \Phi, \mathrm{pc} \vdash \mathrm{e}_{\text {size }} \Downarrow \mathrm{v} \\
& \text { st } \leq \text { st }+ \text { off }<\text { end } \quad \mathrm{v} \in \mathbb{Z}^{+} \quad \text { nalloc }-\mathrm{v}>\nabla \quad \text { nalloc }{ }^{\prime}=\text { nalloc }-\mathrm{v} \\
& \text { Mem }^{\prime}=\operatorname{Mem}[\text { st }+ \text { off } \mapsto(\delta, \text { nalloc }, \text { nalloc, } 0)]\left[a \mapsto 0 \mid a \in\left[\text { nalloc }^{\prime}, \text { nalloc }\right)\right] \\
& \mathrm{Fd}, \mathrm{MVar}, \beta, \Delta, \Sigma, \nabla \vdash\langle\text { Mem, } \Phi, \mathrm{pc} \text {, stk, nalloc }\rangle \rightarrow\left\langle\mathrm{Mem}^{\prime}, \Phi, \text { inc (pc), stk, nalloc' }\right\rangle
\end{aligned}
$$


Fig. 7: (Excerpt) Evaluation of expressions e in CHERIExpress. The evaluation relation $\Downarrow \subseteq \mathrm{e} \times \mathrm{V}$ is defined on pairs of expressions e and values $\mathrm{V}$. The evaluation relation $\Downarrow$ is indexed with an evaluation context Mem, ddc, stc, pcc which is part of an execution state.

(Eval-ddc)

Mem, ddc, stc, pcc $\vdash$ ddc $\Downarrow$ ddc
(Eval-deref)

Mem, ddc, stc, pcc $\vdash$ e $\Downarrow(\delta$, st, end, off $) \quad$ st $\leq$ st + off $<$ end

Mem, ddc, stc, pcc $\vdash \operatorname{deref}(\mathrm{e}) \Downarrow \operatorname{Mem}(\mathrm{st}+$ off $)$

(Eval-inc)

Mem, ddc, stc, pcc $\vdash \mathrm{e} \Downarrow$ (x, st, end, off $) \quad$ Mem, ddc, stc, pcc $\vdash \mathrm{e}_{\mathrm{z}} \Downarrow \mathrm{v}_{\mathrm{z}} \quad \mathrm{v}_{\mathrm{z}} \in \mathbb{Z}$

Mem, ddc, stc, pcc $\vdash \operatorname{inc}\left(e, e_{z}\right) \Downarrow\left(x, s t\right.$, end, off $\left.+\mathrm{v}_{\mathrm{z}}\right)$

$\mathrm{s}_{\mathrm{s}} \cong_{\mathrm{p}} \mathrm{s}_{\mathrm{t}} \wedge\left(\mathrm{s}_{\mathrm{t}}, \varsigma\right) \stackrel{\alpha}{\longrightarrow} \llbracket \mathrm{p} \rrbracket\left(\mathrm{s}_{\mathrm{t}}^{\prime}, \varsigma^{\prime}\right) \Longrightarrow \exists \mathrm{s}_{\mathrm{s}}^{\prime} \cdot\left(\mathrm{s}_{\mathrm{s}}, \varsigma\right) \stackrel{\alpha}{\longrightarrow} \stackrel{\mathrm{p}}{\mathrm{p}}_{\left(\mathrm{s}_{\mathrm{s}}^{\prime}, \varsigma^{\prime}\right) \wedge}$ $\mathrm{s}_{\mathrm{s}}^{\prime} \cong_{\mathrm{p}} \mathrm{s}_{\mathrm{t}}^{\prime}$

Lemma 9 (Lock-step simulation of strong similarity). $\left(\mathrm{s}_{1}, \varsigma\right) \quad \approx_{\llbracket \mathrm{p} \rrbracket}\left(\mathrm{s}_{2}, \varsigma\right) \wedge\left(\mathrm{s}_{1}, \varsigma\right) \stackrel{\tau}{\square} \llbracket \mathrm{p} \rrbracket_{\tau}\left(\mathrm{s}_{1}^{\prime}, \varsigma\right)$

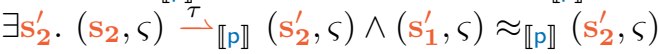

Lemma 10 (Strong similarity is weakened by an output action). $\lambda \in i \wedge\left(\mathrm{s}_{1}, \varsigma\right) \approx_{\llbracket p \rrbracket}\left(\mathrm{s}_{2}, \varsigma\right) \wedge\left(\mathrm{s}_{1}, \varsigma\right) \stackrel{\lambda}{\llbracket p \rrbracket}\left(\mathrm{s}_{1}^{\prime}, \varsigma^{\prime}\right) \Longrightarrow$ $\exists \mathrm{s}_{2}^{\prime} .\left(\mathrm{s}_{2}, \varsigma\right) \stackrel{\lambda}{\llbracket \mathrm{p} \rrbracket}\left(\mathrm{s}_{2}^{\prime}, \varsigma^{\prime}\right) \wedge\left(\mathrm{s}_{1}^{\prime}, \varsigma^{\prime}\right) \sim_{\llbracket \mathrm{p} \rrbracket}\left(\mathrm{s}_{2}^{\prime}, \varsigma^{\prime}\right)$

Lemma 11 (Option simulation of weak similarity). $\left(\mathrm{s}_{1}, \varsigma\right) \sim_{\llbracket \mathrm{p} \rrbracket}\left(\mathrm{s}_{2}, \varsigma\right) \wedge\left(\mathrm{s}_{1}, \varsigma\right) \stackrel{\tau}{\llbracket p \rrbracket}\left(\mathrm{s}_{1}^{\prime}, \varsigma\right) \Longrightarrow\left(\mathrm{s}_{1}^{\prime}, \varsigma\right) \sim_{\llbracket p \rrbracket}$ $\left(\mathrm{s}_{2}, \varsigma\right)$

Lemma 12 (Weak similarity is strengthened by aligned input actions).

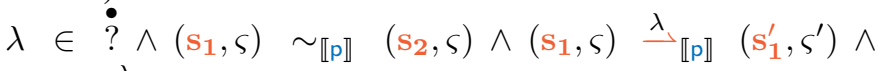
$\left(\mathrm{s}_{2}, \varsigma\right) \stackrel{\lambda}{\llbracket \mathrm{p} \rrbracket}\left(\mathrm{s}_{2}^{\prime}, \varsigma^{\prime}\right) \Longrightarrow\left(\mathrm{s}_{1}^{\prime}, \varsigma^{\prime}\right) \approx_{\llbracket \mathrm{p} \rrbracket}\left(\mathrm{s}_{2}^{\prime}, \varsigma^{\prime}\right)$

Lemma 13 (Adequacy of emulate_invariants). emulate_invariants $(\mathrm{s})_{\alpha, i, \mathrm{p}} \wedge \alpha(i) \in \dot{?} \cup\{\checkmark\} \Longrightarrow$ $\exists \mathrm{s}^{\prime} .\left(\mathrm{s},{ }_{-}\right) \stackrel{\alpha(i)}{\mathrm{p}}\left(\mathrm{s}^{\prime},{ }_{-}\right)$

Lemma 14 (Preservation of emulate_invariants). emulate_invariants $(\mathrm{s})_{\alpha, i, \mathrm{p}} \wedge\left(\mathrm{s},{ }_{-}\right) \stackrel{\alpha(i)}{\mathrm{p}}\left(\mathrm{s}^{\prime},{ }_{-}\right) \Longrightarrow$ emulate_invariants $\left(\mathrm{s}^{\prime}\right)_{\alpha, i+1, \mathrm{p}}$

Using the lemmas above, one can prove that $\operatorname{Tr} I C L$ satisfies step-wise backward-simulation (Lemma 6).

\section{Output of the source-to-source transformation}

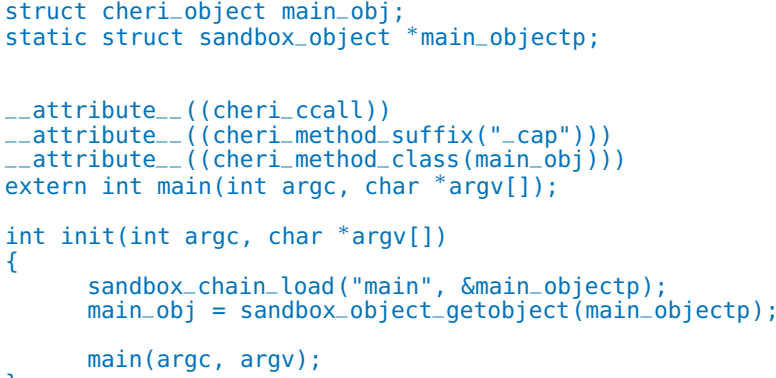

Listing A.1: Source-to-source compilation output. Initialization module init.c

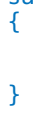

Listing A.2: Source-to-source compilation output. Transformed main.c

extern struct cheri object libl;

struct cheri_object lib2;

-_attribute_- ( (cheri_ccallee)

_-_attribute__( (cheri_method_class (lib1)))

int $f 1$ (void):

attribute ((cheri ccall))

-attribute_- ((cheri_method_class (lib2)))

int $\mathrm{f} 2$ (void);

_-attribute_-_ ((constructor)) static void

sandboxes_init (void)

\{

lib2 = fetch_object ("lib2");

int $f 1$ (void)

$\mathrm{f2}() ;$

Listing A.3: Source-to-source compilation output.

Transformed lib1.c

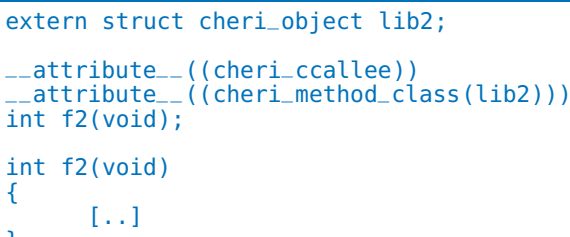

Listing A.4: Source-to-source compilation output. Transformed lib2.c 


\section{REFERENCES}

[1] R. N. Watson, P. G. Neumann, and S. W. Moore, "Balancing disruption and deployability in the cheri instruction-set architecture (isa)," 2017.

[2] J. Woodruff, R. N. Watson, D. Chisnall, S. W. Moore, J. Anderson, B. Davis, B. Laurie, P. G. Neumann, R. Norton, and M. Roe, "The CHERI Capability Model: Revisiting RISC in an Age of Risk," SIGARCH Comput. Archit. News, vol. 42, no. 3, pp. 457-468, Jun. 2014. [Online]. Available: http://doi.acm.org/10.1145/2678373.2665740

[3] R. N. M. Watson, R. M. Norton, J. Woodruff, S. W. Moore, P. G. Neumann, J. Anderson, D. Chisnall, B. Davis, B. Laurie, M. Roe, N. H. Dave, K. Gudka, A. Joannou, A. T. Markettos, E. Maste, S. J. Murdoch, C. Rothwell, S. D. Son, and M. Vadera, "Fast protectiondomain crossing in the cheri capability-system architecture," IEEE Micro, vol. 36, no. 5, pp. 38-49, Sept 2016.

[4] R. N. Watson, J. Woodruff, P. G. Neumann, S. W. Moore, J. Anderson, D. Chisnall, N. Dave, B. Davis, K. Gudka, B. Laurie et al., "CHERI: A hybrid capability-system architecture for scalable software compartmentalization," in Security and Privacy (SP), 2015 IEEE Symposium on. IEEE, 2015, pp. 20-37.

[5] D. Chisnall, C. Rothwell, R. N. Watson, J. Woodruff, M. Vadera, S. W. Moore, M. Roe, B. Davis, and P. G. Neumann, "Beyond the pdp-11: Architectural support for a memory-safe c abstract machine," in ACM SIGPLAN Notices, vol. 50, no. 4. ACM, 2015, pp. 117-130.

[6] S. Tsampas, D. Devriese, and F. Piessens, "Temporal safety for stack allocated memory on capability machines," 2019. [Online]. Available: https://lirias.kuleuven.be/retrieve/538854

[7] L. Skorstengaard, D. Devriese, and L. Birkedal, "Reasoning about a machine with local capabilities," in European Symposium on Programming. Springer, 2018, pp. 475501.

[8] —, "Stktokens: Enforcing well-bracketed control flow and stack encapsulation using linear capabilities," Proc. ACM Program. Lang., vol. 3, no. POPL, pp. 19:1-19:28, Jan. 2019. [Online]. Available: http://doi.acm.org/10.1145/3290332

[9] D. Chisnall, B. Davis, K. Gudka, D. Brazdil, A. Joannou, J. Woodruff, A. T. Markettos, J. E. Maste, R. Norton, S. Son, M. Roe, S. W. Moore, P. G. Neumann, B. Laurie, and R. N. Watson, "CHERI JNI: Sinking the Java Security Model into the C," in International Conference on Architectural Support for Programming Languages and Operating Systems. ACM, 2017, pp. 569-583.

[10] M. Abadi, "Protection in programming-language translations," in International Colloquium on Automata, Languages, and Programming. Springer, 1998, pp. 868-883.

[11] R. N. M. Watson, P. G. Neumann, J. Woodruff, M. Roe, H. Almatary, J. Anderson, J. Baldwin, D. Chisnall, B. Davis, N. W. Filardo, A. Joannou,
B. Laurie, A. T. Markettos, S. W. Moore, S. J. Murdoch, K. Nienhuis, R. Norton, A. Richardson, P. Rugg, P. Sewell, S. Son, and H. Xia, "Capability Hardware Enhanced RISC Instructions: CHERI Instruction-Set Architecture (Version 7)," University of Cambridge, Computer Laboratory, Tech. Rep. UCAM-CL-TR-927, 2019. [Online]. Available: https:/www.cl.cam.ac.uk/techreports/UCAM-CL-TR-927.pdf

[12] R. Milner and R. Weyhrauch, "Proving compiler correctness in a mechanized logic," Machine Intelligence, vol. 7, no. 3, pp. 51-70, 1972.

[13] M. Patrignani, A. Ahmed, and D. Clarke, "Formal approaches to secure compilation: A survey of fully abstract compilation and related work," ACM Comput. Surv., vol. 51, no. 6, pp. 125:1-125:36, Feb. 2019. [Online]. Available: http://doi.acm.org/10.1145/3280984

[14] X. Leroy, "Formal verification of a realistic compiler," Communications of the ACM, vol. 52, no. 7, pp. 107115, 2009.

[15] D. PATTERSON and A. AHMED, "The next 700 compiler correctness theorems (functional pearl)," 2019.

[16] P. Agten, R. Strackx, B. Jacobs, and F. Piessens, "Secure compilation to modern processors," in CSF '12. IEEE, 2012, pp. 171 - 185. [Online]. Available: http://dx.doi.org/10.1109/CSF.2012.12

[17] M. Patrignani, D. Devriese, and F. Piessens, "On Modular and Fully-Abstract Compilation," in Proceedings of the 29th IEEE Computer Security Foundations Symposium CSF 2016, Lisbon, Portugal, ser. CSF 2016, 2016.

[18] M. Patrignani, P. Agten, R. Strackx, B. Jacobs, D. Clarke, and F. Piessens, "Secure compilation to protected module architectures," ACM Transactions on Programming Languages and Systems (TOPLAS), vol. 37, no. 2, p. 6, 2015.

[19] Y. Juglaret and C. Hritcu, "Secure compilation using micro-policies," 2015.

[20] R. Milner, Communicating and mobile systems: the pi calculus. Cambridge university press, 1999.

[21] J. Laird, A fully abstract trace semantics for general references, ser. Lecture Notes in Computer Science (including subseries Lecture Notes in Artificial Intelligence and Lecture Notes in Bioinformatics). Springer Verlag, 7 2007, pp. 667-679.

[22] Y. Juglaret, C. Hriţcu, A. Azevedo de Amorim, and B. C. Pierce, "Beyond good and evil: Formalizing the security guarantees of compartmentalizing compilation," in 29th IEEE Symposium on Computer Security Foundations (CSF). IEEE Computer Society Press, Jul. 2016. [Online]. Available: http://arxiv.org/abs/1602.04503

[23] C. Abate, A. Azevedo de Amorim, R. Blanco, A. N. Evans, G. Fachini, C. Hritcu, T. Laurent, B. C. Pierce, M. Stronati, and A. Tolmach, "When good components go bad: Formally secure compilation despite dynamic compromise," CoRR, vol. abs/1802.00588v2, 2018. [Online]. Available: http://arxiv.org/abs/1802.00588v2

[24] R. M. Norton, D. Chisnall, B. Davis, W. Koszek, 
S. W. Moore, S. J. Murdoch, P. G. Neumann, and J. Woodruff, "Capability Hardware Enhanced RISC Instructions: CHERI Programmer's Guide," University of Cambridge, Computer Laboratory, Tech. Rep. UCAM-CL-TR-877, Sep. 2015. [Online]. Available: http://www.cl.cam.ac.uk/techreports/UCAM-CL-TR-877.pdf

[25] M. A. Jean-loup Gailly, "zlib 1.2.11," 2017. [Online]. Available: https://zlib.net/

[26] K. Simonov, "Libyaml 0.1.7,” 2017. [Online]. Available: https://pyyaml.org/wiki/LibYAML

[27] A. Rubini, “Gnu-barcode 0.99,” 2013. [Online]. Available: https://www.gnu.org/software/barcode/

[28] G. R.-P. Guy Eric Schalnat, Andreas Eric Dilger, “libpng 1.6.34," 2017. [Online]. Available: http://www.libpng.org/pub/png/libpng.html

[29] R. N. M. Watson, P. G. Neumann, J. Woodruff, M. Roe, J. Anderson, J. Baldwin, D. Chisnall, B. Davis, A. Joannou, B. Laurie, S. W. Moore, S. J. Murdoch, R. Norton, S. Son, and H. Xia, "Capability Hardware Enhanced RISC Instructions: CHERI Instruction-Set Architecture (Version 6)," University of Cambridge, Computer Laboratory, Tech. Rep. UCAM-CL-TR-891, 2016. [Online]. Available: https://www.cl.cam.ac.uk/techreports/UCAM-CL-TR-891.pdf

[30] Q. Ge, Y. Yarom, D. Cock, and G. Heiser, "A survey of microarchitectural timing attacks and countermeasures on contemporary hardware," J. Cryptographic Engineering, vol. 8, no. 1, pp. 1-27, 2018.

[31] V. D'Silva, M. Payer, and D. Song, "The correctnesssecurity gap in compiler optimization," in 2015 IEEE Security and Privacy Workshops. IEEE, 2015, pp. 7387.

[32] G. Barthe, B. Grégoire, and V. Laporte, "Secure compilation of side-channel countermeasures: the case of cryptographic "constant-time"," in 2018 IEEE 31 st Computer Security Foundations Symposium (CSF). IEEE, 2018, pp. 328-343.

[33] F. Besson, A. Dang, and T. Jensen, "Securing compilation against memory probing," in Proceedings of the 13th Workshop on Programming Languages and Analysis for Security. ACM, 2018, pp. 29-40.

[34] C. Watt, J. Renner, N. Popescu, S. Cauligi, and D. Stefan, "Ct-wasm: type-driven secure cryptography for the web ecosystem," Proceedings of the ACM on Programming Languages, vol. 3, no. POPL, p. 77, 2019.

[35] T. C. Murray and P. C. van Oorschot, "BP: formal proofs, the fine print and side effects," in 2018 IEEE Cybersecurity Development, SecDev 2018, Cambridge, MA, USA, September 30 - October 2, 2018, 2018, pp. 1-10. [Online]. Available: https://doi.org/10.1109/SecDev.2018.00009

[36] M. Patrignani, "The tome of secure compilation: Fully abstract compilation to protected modules architectures," 2015.

[37] C. Fournet, N. Swamy, J. Chen, P.-E. Dagand, P.-Y. Strub, and B. Livshits, "Fully abstract compilation to javascript," SIGPLAN Not., vol. 48, no. 1, pp. 371-384, Jan. 2013. [Online]. Available: http://doi.acm.org/10.1145/2480359.2429114

[38] M. S. New, W. J. Bowman, and A. Ahmed, "Fully abstract compilation via universal embedding," in Proceedings of the 21st ACM SIGPLAN International Conference on Functional Programming, ser. ICFP 2016. New York, NY, USA: ACM, 2016, pp. 103-116. [Online]. Available: http://doi.acm.org/10.1145/2951913.2951941

[39] D. Devriese, M. Patrignani, and F. Piessens, "Fullyabstract compilation by approximate back-translation," in Proceedings of the 43rd Annual ACM SIGPLANSIGACT Symposium on Principles of Programming Languages, POPL 2016, St. Petersburg, FL, USA, January 20 - 22, 2016, 2016, pp. 164-177. [Online]. Available: http://doi.acm.org/10.1145/2837614.2837618

[40] K. Crary, "Fully abstract module compilation," Proc. ACM Program. Lang., vol. 3, no. POPL, pp. 10:1-10:29, Jan. 2019. [Online]. Available: http://doi.acm.org/10.1145/3290323

[41] C. Abate, R. Blanco, D. Garg, C. Hritcu, M. Patrignani, and J. Thibault, "Journey beyond full abstraction: Exploring robust property preservation for secure compilation," 2018.

[42] M. Patrignani and D. Garg, "Robustly safe compilation," in Programming Languages and Systems, L. Caires, Ed. Cham: Springer International Publishing, 2019, pp. 469498.

[43] — - "Secure Compilation and Hyperproperties Preservation," in Proceedings of the 30th IEEE Computer Security Foundations Symposium CSF 2017, Santa Barbara, USA, ser. CSF 2017, 2017. 